\title{
GEOMETRY OF IMMERSIONS. II
}

\author{
BY \\ E. A. FELDMAN( $\left.{ }^{1}\right)$
}

Introduction. This paper is the natural sequel to Geometry of immersions. I [4]. The principal aim of the two papers as a whole is to sufficiently alter the structure of the theory of singularities of smooth maps of Thom and Whitney, so as to be applicable to some problems concerning geometrically defined singularities, which depend upon particular choices of affine or Riemannian connections. Then having so altered this branch of differential topology, to apply our general results to some specific problems. In [4] we developed an analog of the Thom-Whitney transversality theory, which was applicable to $p$ th order osculating maps defined with respect to a given set of "generalized connections." We then used the transversality theory to prove $p$ th order analogs to the embedding, immersion, isotopy and regular homotopy theorems of Whitney (see [16]). Having developed this analog of the transversality theory, it is natural to see whether we can prove an analog of Thom's polynomial theorem (see Séminaire Cartan 56-57, Exposé 8). Part I of this paper deals with this question. It consists in formally extending the concept of a singularity of a homomorphism between vector bundles to take into account reductions of structural groups which will be given by "geometric" structures on our manifolds. With these geometric reductions built into the theory, a very general existence theorem of the Thom type is stated and proved (Theorems 2.5 and 2.6). The proof is a reworking with more details of the basic ideas in Thom's proof as presented by Haefliger in the Cartan seminar. (See Exposé 8, Séminaire Cartan 56-57). However some pains are taken to insure that the gaps in the earlier presentation (i.e., the whole question of fundamental classes) are not present in this one. With the recent work of Borel, Moore, and Haefliger, (see [1] and [2]), these gaps are filled.

Finally, in Part II we apply this machinery to certain classical problems in extrinsic differential geometry. The first section relates the maps $D_{2} T_{2}(f)$ to second fundamental forms in the case where $f$ is an immersion. It thereby gives us some geometric insight into what the "nondegenerate" $D_{2} T_{2}(f)$ of [4] were about. In the last two sections we apply this theory to problems concerning inflection points and totally geodesic points.

A more complete outline of this paper has appeared as a research announcement

Received by the editors February 8, 1965.

(1) This research was supported by the following contracts: NONR-266(57) and NSF 19022 at Columbia University and NSF GP-2425 at Princeton University. Part of this paper is contained in the author's doctoral dissertation at Columbia University. 
in the July 1964 issue of the Bull. Amer. Math. Soc. However, we have changed the words " $p$ th order symmetric linear connection" to " $p$ th order dissection" in accordance with [4].

Much of the material in this paper was contained in the author's doctoral dissertation at Columbia University. Hence I would like to thank again J. Eells, W. Pohl, and I. Porteous for their interest and encouragement. However the last part of $\S 7$ is new.

The material about parabolic and umbilic points will appear in a sequel shortly to appear. The reason for this is the study of these singularities involves a detailed study of the "singular varieties" in question, as well as the general theory, whereas the inflection and totally geodesic sections are rather direct applications of the general theory.

\section{0 . Notations and conventions.}

a. All manifolds discussed in this paper will be finite dimensional and all the connected components of a given manifold will have the same dimension. The manifolds will satisfy the second axiom of countability and will be of class $C^{\infty}$. We will use the adjectives smooth or differentiable to connote a manifold, or a map between manifolds of class $C^{\infty}$.

b. If $X$ and $Y$ are smooth manifolds we will denote by $C^{k}(X, Y)$ the set of smooth maps from $X$ to $Y$ with the "fine" $C^{k}$-topology, and $C(X, Y)$ the set of smooth maps with the fine $C^{\infty}$-topology. This topology is merely the union of all the open sets of the $C^{k}$-topologies. It is a topology because it coincides with the inverse limit topology on $C(X, Y)$ viewing the $C^{k}(X, Y)$ with the obvious inclusion maps as an inverse mapping system. For further details on this topology we refer the reader to the appendix at the end of [4]. This appendix includes a proof of the fact that $C(X, Y)$ is a Baire space in the fine topology. All the theorems in this paper are stated in their " $C^{\infty}$." form; it is clear that they are also true " $C^{k}$ " for $k$ sufficiently large. The $k$ can be determined by counting the number of derivatives used, and comparing that number with the restrictions imposed by the Sard theorem.

c. If $\xi=(\pi: E \rightarrow X)$ is a smooth vector bundle we will denote by $S(\xi)$ the space of smooth sections of $\xi$ topologized as a subset of $C(X, E)$. We will denote by $F(X)$ the space of smooth real valued functions $C(X, R)$. We will also denote the total space of the tangent vector bundle over a smooth manifold $X$ by $T_{1}(X)$.

d. If $\xi=(\pi: E \rightarrow X)$ is a smooth vector bundle, we will mean by a linear (affine) connection on $\xi$ a covariant derivative, i.e., a map

$$
D: S\left(T_{1}(X)\right) \times S(E) \rightarrow S(E)
$$

such that

(1) $D_{f x+g y} e=f D_{x} e+g D_{y} e$ for every $e \in S(E), f, g \in F(X)$ and $x, y \in S\left(T_{1}(X)\right)$. 
(2) $D_{x} f e=f D_{x} e+x(f) e$ for every $x \in S\left(T_{1}(X)\right), e \in S(E), f \in F(X)$ and $x(f)$ is evaluation of the vector field $x$ on the function $f$.

e. If $X$ is a smooth manifold denote by $F$ the sheaf of germs of smooth functions on $X$. If $U \subset X$ let $\Gamma(U, \quad)$ represent the section functor for sheaves. Let $F(U)=$ $\Gamma(U, F)$. If $\xi=(\pi: E \rightarrow X)$ is a smooth vector bundle over $X$, let $\mathbf{E}$ be the sheaf of germs of smooth cross-sections of $\xi$. $\mathbf{E}$ is a sheaf of $\mathbf{F}$-modules. It is clear that $\Gamma(U, E)$ is an $F(U)$ module and that $\Gamma(X, \mathbf{E})=S(E)$ and is an $F(X)$ module. For a full account of this "dialogue" between smooth vector bundles and their sheaves of germs of smooth sections we refer the reader to Koszul's notes (Koszul [15]).

f. Let $\xi=(\pi: E \rightarrow X)$ and $\eta=\left(\pi^{\prime}: E^{\prime} \rightarrow Y\right)$ be smooth vector bundles. Denote by $\operatorname{Hom}(\xi, \eta)$ (or by an abuse of notation $\operatorname{Hom}\left(E, E^{\prime}\right)$ ) the vector bundle over $X \times Y$ whose fiber over the point $(x, y) \in X \times Y$ is the vector space $\operatorname{Hom}\left(E_{x}, E_{y}^{\prime}\right)$. This set is made into a vector bundle in the obvious way. Let $D: X \rightarrow X \times X$ be the diagonal map, and let $\xi=(\pi: E \rightarrow X)$ and $\eta=\left(\pi^{\prime}: E^{\prime} \rightarrow X\right)$ be smooth vector bundles. We shall denote by $\operatorname{Hom}\left(E, E^{\prime}\right) \mid X$ the bundle $D^{-1}\left(\operatorname{Hom}\left(E, E^{\prime}\right)\right)$ where $D^{-1}$ ( ) means the "pull back" of the bundle over $X \times X$, to a bundle over $X$. This latter bundle (the one we denoted by $\left.\operatorname{Hom}\left(E, E^{\prime}\right) \mid X\right)$ is the bundle more usually denoted by $\operatorname{Hom}\left(E, E^{\prime}\right)$.

g. The following number appears very frequently in our work, so we have set aside a special notation for it. Let

$$
v(n, p)=n+\frac{n(n+1)}{2}+\cdots+\frac{n(n+1)(n+2)(\cdots)(n+p-1)}{p !} .
$$

h. Let $\xi=(\pi: E \rightarrow X)$ be a smooth vector bundle. Then we denote by $O^{p} E$ the $p$-fold symmetric tensor product of $E$ with itself. Let $\eta=\left(\pi^{\prime}: E^{\prime} \rightarrow Y\right)$ be another vector bundle. We connote by the expression " $g: E \rightarrow E^{\prime}$ is a vector bundle homomorphism covering $f: X \rightarrow Y$ " the commutative diagram of smooth maps below, where $g$ maps each fiber $E_{x}$ linearly into the fiber $E_{f(x)}^{\prime}$.

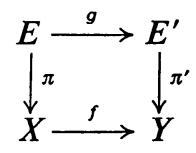

i. Let $X$ be a smooth manifold. Let $N$ be a submanifold of $X$, and let $i: N \rightarrow X$ be the inclusion map. We say that $N$ is a regular submanifold if $i$ is a diffeomorphism into.

j. Let $X$ be a smooth manifold, and let $\mathfrak{A}=\left(\varphi_{i}, U_{i}\right) i=1,2, \ldots$ be a countable atlas for $X$. We say that $\mathfrak{A}$ is an admissible atlas if

1. $\varphi_{i}\left(U_{i}\right) \cong R^{n}$

2. the open unit balls $B_{i}\left(B_{i}=\varphi_{i}^{-1}\right.$ (unit ball in $\left.R^{n}\right)$ ) cover $X$;

3. the covering $\left(U_{i}\right), i=1,2, \ldots$ is locally finite. 
k. Let $X$ be a smooth manifold. We will denote by $T_{p}(X)$, the $p$ th order tangent bundle of $X$. If $f \in \mathscr{C}(X, Y)$ we denote by $T_{p}(f)$ the $p$ th differential of $f$, (see $[4, \S 1]$ ). Let $\left(D^{(k)}\right), k=1,2, \ldots$ be a sequence of $k$ th order dissections on $Y$. We will denote by $D_{p} T_{p}(f)=D^{1} D^{2} \cdots D^{p-1} T_{p}(f)$, the $p$ th order osculating map of $f$ with respect to the dissections $\left(D^{k}\right)$. Finally, with respect to a fixed set of dissections $\left(D^{k}\right)$, we denote by $I^{p}(X, Y)$ and $E^{p}(X, Y)$ respectively, the set of $p$ th order immersions of $X$ in $Y$ and the set of $p$ th order embeddings of $X$ in $Y$, (see $[4, \S 1-\S 3])$.

\section{Part I - Singularity Theory}

The aim of this part is twofold. The first is to state and prove a generalization of the Thom Polynomial Theorem, (see [12]). The second is to show that some explicit formulas for Thom polynomials due to I. R. Porteous (see [8]) can be applied under hypotheses slightly different than Porteous'.

$N . B$. In $\S \S 1,2$, and 4 the manifolds denoted by the letters $X$ and $Y$ will be assumed to be connected, unless it is explicitly stated to the contrary.

1. $G$-structures on vector bundles, their singularities and characteristic classes. This section will consist of definitions to extend the concept of a singularity of a map between vector bundles.

Definition 1.1. Let $X$ be a smooth manifold. A submanifold collection $K=$ $K_{1} \cup K_{2} \cup \cdots \cup K_{s}$ of submanifolds of $X$ is a finite collection of disjoint submanifolds $K_{i}$ of $X$ such that;

(a) $\operatorname{Dim} K_{i-1}>\operatorname{Dim} K_{i}$ and

(b) $K_{i} \cup \cdots \cup K_{s}$ is closed in $X$. The points of $K_{1}$ will be called the regular points. The dimension of $K$ will always mean the dimension of $K_{1}$.

Definition 1.2. Let $\varphi_{1}: G \rightarrow G L(p, R)$ and $\varphi_{2}: H \rightarrow G L(q, R)$ be faithful representations of Lie groups $G$ and $H$ in $G L(p, R)$ and $G L(q, R)$ respectively. This induces an effective action of $G \times H$ on $\operatorname{Hom}\left(R^{p}, R^{q}\right)$. Let $K$ be a regular submanifold collection of $\operatorname{Hom}\left(R^{p}, R^{q}\right)$. Then $K$ is called a model $G \times H$ singularity with respect to the representations $\varphi_{1}$ and $\varphi_{2}$ if $K$ is invariant under the action of $G \times H$ on $\operatorname{Hom}\left(R^{p}, R^{q}\right)$.

Definition 1.3. Let $\varphi_{1}: G \rightarrow G L(p, R)$ and $\varphi_{2}: H \rightarrow G L(q, R)$ be faithful representations of the Lie groups $G$ and $H$ respectively. Let $K \subseteq \operatorname{Hom}\left(R^{p}, R^{q}\right)$ be a model $G \times H$ singularity with respect to the representations $\varphi_{1}$ and $\varphi_{2}$. Let $B_{G}^{n+1}$ and $B_{H}^{n+1}$ be the $n+1$ universal classifying space for $G$-bundles and the $n+1$ universal classifying space for $H$-bundles respectively. It is clear that $B_{G}^{n+1} \times B_{H}^{n+1}$ is an $n+1$ universal classifying space for $G \times H$ bundles. Let $\widetilde{E}^{n+1}$ be the vector bundle with fiber Hom $\left(R^{p}, R^{q}\right)$ over $B_{G}^{n+1} \times B_{H}^{n+1}$ associated to the $n+1$ universal principal $G \times H$ bundle $\pi_{G}^{n+1} \times \pi_{H}^{n+1}: E_{G}^{n+1} \times E_{H}^{n+1} \rightarrow B_{G}^{n+1} \times B_{H}^{n+1}$, by the action of $G \times H$ on Hom $\left(R^{p}, R^{q}\right)$ induced by the representations $\varphi_{1}$ and $\varphi_{2}$. The model $G \times H$ singularity $K \subseteq \operatorname{Hom}\left(R^{p}, R^{q}\right)$ then induces a submanifold collection $K_{\tilde{E}^{n+1}}$ of $\widetilde{E}^{n+1} . K_{\tilde{E}^{n+1}}$ is itself a fiber bundle with structural group $G \times H$, fiber $K$ and base 
$B_{G}^{n+1} \times B_{H}^{n+1} . K_{\tilde{E}^{n+1}}$ is called the $n+1$ universal singularity, given by the model $G \times H$ singularity $K$ (and the representations $\varphi_{1}$ and $\varphi_{2}$ ).

DEFINITION 1.4. Let $\xi=(\pi: E \rightarrow X)$ be a smooth vector bundle of fiber dimension $q$. Let $\pi_{E}: P_{E} \rightarrow X$ be the principal $G L(q, R)$ bundle associated to $E$. Let $G$ be a Lie group. Let $\varphi: G \rightarrow G L(q, R)$ be a faithful representation of $G$. A $(G, \varphi)$ structure on $E$ is given by a principal $G$-bundle $\pi_{G}: P_{G} \rightarrow X$, and a mapping $\tilde{\varphi}: P_{G} \rightarrow P_{E}$ such that;

(a) $\pi_{E} \cdot \tilde{\varphi}=\pi_{G}$,

(b) $\tilde{\varphi}$ is smooth and $1-1$, and

(c) for each $u \in P_{G}$ and $g \in G, \tilde{\varphi}(u) \varphi(g)=\tilde{\varphi}(u g)$.

Remark. A $(G, \varphi)$ structure on a vector bundle $\xi=\{\pi: E \rightarrow X\}$ of fiber dimension $q$, gives a reduction of the structural group $G L(q, R)$ to $\varphi(G)$. Hence the representation $\varphi$ allows us to consider the vector bundle $\xi$ as a vector bundle associated with the principal $G$-bundle $P_{G}$, because the $G$ action on the fibers will be linear.

DEFINITION 1.5. Let $\pi: E \rightarrow X$, and $\pi^{\prime}: E^{\prime} \rightarrow Y$ be smooth vector bundles of fiber dimension $p$ and $q$ respectively. Let there be given a $\left(G, \varphi_{1}\right)$ structure on $E$ and an $\left(H, \varphi_{2}\right)$ structure on $E^{\prime}$. Let $K$ be a model $G \times H$ singularity with respect to the representations $\varphi_{1}$ and $\varphi_{2}$. The $\left(G, \varphi_{1}\right)$ structure on $E$ and the $\left(H, \varphi_{2}\right)$ structure on $E^{\prime}$ induce a $\left(G \times H, \varphi_{1}^{\operatorname{tr}^{-1}} \times \varphi_{2}\right)$ structure on Hom $\left(E, E^{\prime}\right)$. Hence we can consider Hom $\left(E, E^{\prime}\right)$ as a vector bundle with fiber Hom $\left(R^{p}, R^{q}\right)$ structural group $G \times H$, and base $X \times Y$. Due to the fact that $K$ is invariant under the $G \times H$ action induced on $\operatorname{Hom}\left(R^{p}, R^{q}\right), K$ defines a regular submanifold collection $K(X, Y)$ of Hom $\left(E, E^{\prime}\right)$. Let $K=K_{1} \cup \cdots \cup K_{s}$, then $K(X, Y)=K_{1}(X, Y) \cup \cdots \cup$ $K_{s}(X, Y)$ where codim $\left(K_{i}\right)=\operatorname{codim}\left(K_{i}(X, Y)\right)$. Furthermore $K(X, Y)\left(K_{i}(X, Y)\right)$ is a fiber bundle over $X \times Y$, with structural group $G \times H$ and fiber $K,\left(K_{i}\right) . K(X, Y)$ is called the $G \times H$ singularity defined by the $\left(G, \varphi_{1}\right)$, structure on $E$, the $\left(H, \varphi_{2}\right)$ structure on $E^{\prime}$, and the model $G \times H$ singularity with respect to $\varphi_{1}, \varphi_{2}$, and $K$.

Putting these definitions together yields the following result.

Proposition 1.6. Let $\pi: E \rightarrow X$ and $\pi^{\prime}: E^{\prime} \rightarrow Y$ be smooth vector bundles. Let $\operatorname{Dim}(X)=n$ and $\operatorname{Dim}(Y)=N$. Let there be given $a\left(G, \varphi_{1}\right)$ structure on $E$ and an $\left(H, \varphi_{2}\right)$ structure on $E^{\prime}$. Let $K$ be a model $G \times H$ singularity with respect to $\varphi_{1}$ and $\varphi_{2}$. Let $\pi_{G \times H}: P_{G \times H} \rightarrow X \times Y$ be the principal $G \times H$ bundle given by the induced $\left(G \times H, \varphi_{1}^{\mathrm{tr}-1} \times \varphi_{2}\right)$ structure on Hom $\left(E, E^{\prime}\right)$. Let $K_{\tilde{E}^{n+N+1}}$ be the $(n+N+1)$-universal singularity given by the model $G \times H$ singularity $K$. Let $f \in\left[X \times Y, B_{G}^{n+N+1} \times B_{H}^{n+N+1}\right]$ be the classifying map for $P_{G \times H}$. Let $K(X, Y)$ be the $G \times H$ singularity defined by the $\left(G, \varphi_{1}\right)$ structure on $E$, the $\left(H, \varphi_{2}\right)$ structure on $E^{\prime}$, and the $G \times H$ singularity $K$.

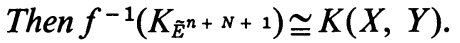

REMARK. Whenever no confusion can arise specific reference to the representations will be dropped. 
Definition 1.7. Let $\pi: E \rightarrow X$ and $\pi^{\prime}: E^{\prime} \rightarrow Y$ be smooth vector bundles. Let there be given a $\left(G, \varphi_{1}\right)$ structure on $E$ and an $\left(H, \varphi_{2}\right)$ structure on $E^{\prime}$. Let $K$ be a model $G \times H$ singularity, and let $K(X, Y)$ be the $G \times H$ singularity defined by the $G$ structure on $E$, the $H$ structure on $E^{\prime}$, and the model $G \times H$ singularity $K$. Let $f: E \rightarrow E^{\prime}$ be a smooth vector bundle homomorphism over the smooth map $\bar{f}: X \rightarrow Y$. This induces a map $\hat{f}: X \rightarrow \operatorname{Hom}\left(E, E^{\prime}\right)$ defined by $\hat{f}(x)=f \mid E_{x^{\prime}} x \in X$. We say $x$ is a $K$-singular point of $f$ if $\hat{f}(x) \in K(X, Y)$. We say that $f$ displays the singularity transversally if $\hat{f}(X) \hbar K(X, Y)$. The set $\hat{f}^{-1}(K(X, Y))=S_{K}(f)$ is called the set of $K$-singular points of $f$. It is clear that if $\hat{f}(X) \hbar K(X, Y)$ then $S_{K}(f)$ is a submanifold collection of the same codimension as $K(X, Y)$.

The foregoing definitions and remarks imply the following proposition.

Proposition 1.8. Let $\pi: E \rightarrow X$ and $\pi^{\prime}: E^{\prime} \rightarrow Y$ be smooth vector bundles. Let there be given a $\left(G, \varphi_{1}\right)$ structure on $E$ and an $\left(H, \varphi_{2}\right)$ structure on $E^{\prime}$. Let $K$ be a model $G \times H$ singularity and let $K(X, Y)$ be the usual $G \times H$ singularity defined by the above structures. Let $f: E \rightarrow E^{\prime}$ be a smooth vector bundle homomorphism over a smooth map $\bar{f}: X \rightarrow Y$. $f$ then induces as usual a vector bundle homomorphism $f !: E \rightarrow \bar{f}^{-1}\left(E^{\prime}\right)$ over $X . K$ then induces in the obvious way a singularity $K(X, X) \subseteq$ $\operatorname{Hom}\left(E, \bar{f}^{-1}\left(E^{\prime}\right)\right)$. Let $\Delta$ be the diagonal map of $X$ in $X \times X$. Let $\Delta^{-1}\left(\operatorname{Hom}\left(E, \bar{f}^{-1}\left(E^{\prime}\right)\right)\right)$ be denoted by $\left.\operatorname{Hom}\left(E, \bar{f}^{-1}\left(E^{\prime}\right)\right)\right|_{X}$. As is well known smooth vector bundle homomorphisms from $E$ into $f^{-1}\left(E^{\prime}\right)$ over $X$ are smooth sections of $\left.\operatorname{Hom}\left(E, f^{-1}\left(E^{\prime}\right)\right)\right|_{X}$ and conversely. Kinduces in the obvious way a submanifold $K(X)$ of $\left.\operatorname{Hom}\left(E, \bar{f}^{-1}\left(E^{\prime}\right)\right)\right|_{X}$, which can also be described by $K(X)=\Delta^{-1}(K(X, X))$. Then $f !(x) \in K(X)$ if and only if $\hat{f}(x) \in K(X, Y)$. Furthermore $f !(X) \pitchfork K(X)$ if and only if $f$ displays the singularity $K$ transversally.

DeFinition 1.9. Let $G$ be a Lie group, and $\xi$ be a smooth principal $G$ bundle over a countable $C W$ complex $X$. Let $B_{G}$ be the universal classifying space for principal $G$ bundles. Let $f \in\left[X, B_{G}\right]$ be the classifying map of $\xi$. Let $A$ be a coefficient ring. The characteristic algebra of $\xi$ with coefficients in $A$ is $f^{*}\left(H^{*}\left(B_{G} ; A\right)\right) \subseteq$ $H^{*}(X ; A)$. The elements of $f^{*}\left(H^{*}\left(B_{G} ; A\right)\right)$ are called the A-characteristic classes of $\xi$, and the map $f^{*}$ is called the characteristic homomorphism of $\xi$.

The following proposition and its corollary will be of use in the next section.

Proposition 1.10. Let $G$ be a Lie group. Let $B_{G}$ be the universal classifying space, and let $B_{G}^{n+1}$ be the $n+1$ universal classifying space for $G$ bundles. Let $A$ be a coefficient ring. Then $H^{i}\left(B_{G}, A\right)=H^{i}\left(B_{G}^{n+1} ; A\right)$ for all $i \leqq n$.

Proof. Let $f: B_{G}^{n+1} \rightarrow B_{G}$ be the classifying map for the $n+1$ universal principal $G$ bundle $\pi_{G}^{n+1}: E_{G}^{n+1} \rightarrow B_{G}^{n+1}$. Then the exact homotopy sequence of a fibration, plus the five lemma yield the fact that $f_{\#}: \pi_{i}\left(B_{G}^{n+1}\right) \rightarrow \pi_{i}\left(B_{G}\right)$ is an isomorphism for 
$0 \leqq i \leqq n$. We then apply Whitehead's theorem to get $f_{*}: H_{i}\left(B_{G}^{n+1}\right) \rightarrow H_{i}\left(B_{G}\right)$ is an isomorphism for $i \leqq n$. Hence our proposition follows from the universal coefficient theorem.

COROLlary 1.11. This enables us to define the A-characteristic classes of a $G$-bundle over a countable $C W$ complex of dimension $n$, by looking at $f^{*}\left(H^{*}\left(B_{G}^{n+1} ; A\right)\right)$ where $f$ is the classifying map of the G-bundle in question.

$N . B$. Let $G$ be a Lie group. We will denote by $G_{c}$ the maximal compact subgroup of $G$. It is well known that $B_{G} \simeq B_{G_{c}}$, and $B_{G}^{n+1} \simeq B_{G_{c}}^{n+1}$.

We will now state some well-known theorems without proofs.

THEOREM 1.12. Let $O(n)$ be the orthogonal group.

(a) There exist classes $W_{i} \in H^{i}\left(B_{O(n)} ; Z_{2}\right)$ called the universal Stiefel-Whitney classes such that $H^{*}\left(B_{O(n)} ; Z_{2}\right) \cong Z_{2}\left[W_{1}, \ldots, W_{n}\right]$. Let $G$ be a Lie group whose maximal compact subgroup $G_{c}$ is $O(n)$. Let $\xi$ be a principal $G$ bundle over a paracompact space $X$. Let $f \in\left[X, B_{G}\right]$ be the classifying map of $\xi$. Then $W_{i}(\xi)=f^{*} W_{i}$ is called the ith Stiefel-Whitney class of $\xi$.

(b) $B_{O(n)}^{m+1}=G_{n, n+m+1}$ the Grassmann manifold of $n$-planes in $n+m+1$ space. $G_{n, n+m+1}$ is a compact manifold of dimension $n(m+1)$. There exist classes $W_{i} \in H^{i}\left(G_{n, n+m+1} ; Z_{2}\right)$ such that $H^{*}\left(B_{G}^{m+1} ; Z_{2}\right)=Z_{2}\left[W_{1}, \ldots, W_{n}\right]$ monomials of degree $>n(m+1)$ where $W_{i}$ is of degree $i$. Let $X$ be a countable $C W$ complex of dimension $\leqq m$. Let $G$ be as in part (a), and let $\xi$ be a G-bundle over $X$. Let $f \in\left[X, B_{G}^{m+1}\right]$ be the classifying map of $\xi$. Then the $f^{*} W_{i}$ are just the Stiefel-Whitney classes $W_{i}(\xi)$ defined in (a).

Definition 1.13. Let $(\pi: E \rightarrow X)=\xi$ be a vector bundle. Let $G$ be a Lie group. Let $\pi_{G}: P_{G} \rightarrow X$ be the principal $G$-bundle given by a $(G, \varphi)$ structure on $E$. Let $A$ be a coefficient ring. The A characteristic classes of the $G$-structure $(G, \varphi)$ on $E$ are defined to be the $A$-characteristic classes of $P_{G}$. If $G_{c}=O(k)$ and if $A=Z_{2}$ characteristic classes of the $G$-structure are just the Stiefel-Whitney classes of $P_{G}$. These will be called the Stiefel-Whitney classes associated with the $(G, \varphi)$ structure on $\xi$. We will denote these classes by $W_{i}^{G}(\xi)$.

Remark. It is clear that the Chern and Pontrjagin classes admit similar discussions.

2. A polynomial theorem. The main result of this section is the statement and proof of a polynomial theorem which is merely a recasting of Thom's Theorem (see Séminaire Cartan 56-57, Exposé 8). Before entering the main discussion we will digress briefly to discuss the Borel-Moore homology theory for locally compact spaces and fundamental classes. 
Throughout this digression we will assume that $X$ is a locally compact and paracompact topological space. We will furthermore assume that all modules and sheaves of modules will be $Z_{2}$-modules, where $Z_{2}$ can denote either itself or the simple sheaf over $X$ with stalk $Z_{2}$. $C$ will always denote the family of compact subsets of $X$. We will first outline the definition of the Borel-Moore groups by following [1]. However, we can make many simplifications because $Z_{2}$ is a field. Therefore the canonical injective resolution of a sheaf of $Z_{2}$-modules and the canonical flasque resolution coincide. If $\mathscr{S}$ is a sheaf of $Z_{2}$-modules let $\mathscr{C} *(X, \mathscr{S})=$ $\left(\mathscr{C}^{p}(X, \mathscr{S})\right)$ denote the canonical flasque resolution of $\mathscr{S}$. If $\Phi$ is a family of supports denote by $C^{*}(X, \mathscr{S})=\Gamma_{\Phi}\left(\mathscr{C}^{*}(X, \mathscr{S})\right)$, the standard cochains of $X$ with coefficients in $\mathscr{S}$ and supports in $\Phi$.

Let $\mathscr{S}$ be a sheaf of $Z_{2}$-modules. Look at the presheaf defined by $U \rightarrow \mathscr{L}(U)$ where

$$
\mathscr{I}(U)=\operatorname{Hom}\left(\Gamma_{C}(\mathscr{S} \mid U), A\right)
$$

where $A$ is a $Z_{2}$-module. Then 2.3 of [1] tells us, if $\mathscr{S}$ is $C$-mou then $U \rightarrow \mathscr{I}(U)$ forms a sheaf, and furthermore the sheaf is flasque.

Definition 2.1. Let $\mathscr{A}=\left(\mathscr{A}^{p}\right)$ be a differential graded sheaf of $Z_{2}$-modules, we denote by $D(\mathscr{A})$ the differential graded sheaf whose $r$ th component is determined by the presheaf $U \rightarrow \operatorname{Hom}\left(\Gamma_{C}\left(\mathscr{A}^{r} \mid U\right), Z_{2}\right)$, with the usual differential. Let $\mathscr{C}_{H}(X)$ denote the differential graded sheaf $D\left(\mathscr{C}^{*}\left(X, \mathbf{Z}_{2}\right)\right)$. If $\mathscr{S}$ is any sheaf of $Z_{2}$-modules we denote by $\mathbf{C}_{H}(X, \mathscr{S})$ the sheaf $\mathscr{S} \otimes \mathbf{C}_{H}(X) . \mathbf{C}_{H}(X)$ and $\mathbf{C}_{H}(X, \mathscr{S})$ are called respectively the standard sheaf for homology on $X$ and the standard sheaf for homology with coefficients in $\mathscr{S}$. If $\Phi$ is any family of supports, we let $C_{H}^{\Phi}(X, \mathscr{S})=$ $\Gamma_{\Phi}\left(\mathbf{C}_{H}(X ; \mathscr{S})\right)$ and $H_{n}^{\Phi}(X, \mathscr{S})=H_{n}\left(C_{H}^{\Phi}(X, \mathscr{S})\right)$. The module $H_{n}^{\Phi}(X ; \mathscr{S})$ is called the $n$-dimensional homology group of $X$ with coefficients in $\mathscr{S}$ and supports in $\Phi$. If $\Phi$ is the family of all closed subsets of $X$ we denote $H_{H}^{\Phi}(X, \mathscr{S})$ by $H_{n}(X, \mathscr{P})$. If $\mathscr{S}=Z_{2}$ we denote $H_{n}^{\Phi}\left(X, \mathbf{Z}_{2}\right)$ by $H_{n}^{\Phi}(X)$. The derived sheaf of $C_{H}(X, \mathscr{S})$, $\mathbf{H}_{*}(X, \mathscr{S})$ is called the local homology sheaf over $X$, and its stalk $\mathbf{H}_{*}(X, \mathscr{S})_{x}$ over $x \in X$, is called the local homology group at $x$. If $\mathscr{S}=Z_{2}$ we denote $\mathbf{H}_{*}(X, \mathscr{S})$ and $\mathbf{H}_{*}(X, \mathscr{S})_{x}$ respectively by $\mathbf{H}_{*}(X)$ and $\mathbf{H}_{*}(X)_{x}$. This homology theory has the following properties that we will make use of in our discussion. For proofs of these properties and a complete account of this theory we again refer the reader to [1].

(1) Let $U$ be an open subset of $X$. Then we have a homomorphism $j_{*}^{X U}: H_{*}(X) \rightarrow$ $H_{*}(U)$ induced by restricting the sections to $U$. Then we have $\mathbf{H}_{*}(X)_{x}=$ $\operatorname{Dir} \lim \left(H_{*}(U) ; j_{*}^{X U}\right)$ where $U$ runs through the neighborhoods of $x$.

(2) If $X$ is a separable metric space of topological dimension $\leqq n$, then there exists a natural isomorphism $\Delta: H_{n}(X) \rightarrow \Gamma\left(X, \mathbf{H}_{n}(X)\right)$.

(3) Let $X$ be an $n$ dimensional topological manifold. Then

$$
\begin{aligned}
\mathbf{H}_{i}(X) & =0, \quad i \neq n . \\
\mathbf{H}_{n}(X) & =\text { the simple sheaf } Z_{2} \times X .
\end{aligned}
$$


(4) Let $F$ be a closed subspace of $X$. Then the inclusion map induces a homomorphism $i_{*_{F} X}: H_{*}(F) \rightarrow H_{*}(X)$. (In general the maps of the category on which these groups are a functor are the proper maps.) We will denote $i_{* F X}$ by $i_{*}$ as there will be no confusion in any of our applications.

(5) The Borel-Moore theory and the Čech theory are one in the same when restricted to the category of compact spaces and continuous maps (actually compact pairs).

(6) Let $X$ be a topological manifold of dimension $n$. Then there exists a Poincaré duality isomorphism $D_{X}: H_{i}(X) \rightarrow H^{n-i}(X)$. The cohomology in question is either Čech, singular, Alexander-Spanier, or canonical (Grothendieck) cohomology, as they are all equivalent in this case.

Definition 2.2. Let $K=K_{1} \cup \cdots \cup K_{s}$ be a submanifold collection of the smooth manifold $X$. Let the dimension of $K=k$. Then by combining properties (1) and (3) of the Borel-Moore groups, we know $\mathbf{H}_{k}(K)_{x}=Z_{2}$ for $x \in K_{1}$. We say $K$ carries a fundamental class $\alpha$ if there exists an element $\alpha \in H_{k}(K)$ such that $\Delta(\alpha)(x)$ is the generator of $\mathbf{H}(K)_{x}, x \in K_{1}$, where $\Delta$ is the map in (2). If such an $\alpha$ exists it is known to be unique by Proposition 2.3 of [2].

REMARK. Unique fundamental classes exist under the following conditions:

(1) If $K=K_{1} \cup \cdots \cup K_{s}$ is a submanifold collection in $X$, and

and

$$
\operatorname{Dim} K_{1}-\operatorname{Dim} K_{2} \geqq 2,
$$

(2) if $X=R^{p}$ and $K$ is a real algebraic variety. The proofs of these statements as well as the two lemmas that follow are to be found in [2].

LemMa 2.3. Let $K$ be a submanifold collection of an $n$ dimensional manifold $X$. Let $Y$ be a smooth $N$ dimensional manifold. Let $\pi: E \rightarrow Y$ be a fiber bundle over $Y$ with fiber $X$, and structural group $G$, such that the action of $G$ on the fiber leaves $K$ invariant. Then $K$ determines a submanifold collection $K(Y) \subseteq E$. Then if $K$ carries a fundamental class so does $K(Y)$.

Lemma 2.4. Let $X$ and $Y$ be smooth manifolds of dimension $n$ and $N$ respectively. Let $f: X \rightarrow Y$ be a smooth map. Let $K=K_{1} \cup \cdots \cup K_{s}$ be a submanifold collection in $Y$. Assume that $f(X) \pitchfork K_{i}$ for all $i$. Let $K^{\prime}=f^{-1}(K)$ and let $i: K \rightarrow Y$ and $j: K^{\prime} \rightarrow X$ be the inclusion maps. Let $D_{X}$ and $D_{Y}$ be Poincaré duality in $X$ and $Y$ respectively. Let us furthermore assume that $K$ is closed in $Y$, and $K_{1}$ is a regular submanifold. If $K$ carries a fundamental class $\alpha$, then $K^{\prime}$ carries a fundamental class $\beta$ such that

$$
f^{*} D_{Y} i_{*} \alpha=D_{X} j_{*} \beta \text {. }
$$

Lemma 2.4 is a direct consequence of 2.3 and 2.12 of [2]. Finally if $K$ is a real algebraic variety, the theorems of Whitney [3] decompose $K$ into a manifold collection $K_{1} \cup \ldots \cup K_{s}$ where $K_{1}$ is a regular submanifold. 
REMARK. This theory as well as this theorem is true for coefficient rings other than $Z_{2}$ (i.e., the integers), under suitable orientability assumptions. However an adequate treatment would require much more French homology theory than I am willing to do at the moment, in that the following theorem is sufficient for all my applications.

THEOREM 2.5. (1) Let $\xi=(\pi: E \rightarrow X)$ and $\eta=\left(\pi: E^{\prime} \rightarrow Y\right)$ be smooth vector bundles of fiber dimension $p$ and $q$ respectively. Let $\xi$ be given $a\left(G, \varphi_{1}\right)$ structure and $\eta$ an $\left(H, \varphi_{2}\right)$ structure respectively. Assume $G_{c}=O(k)$ and $H_{c}=O(t)$.

(2) Let $K$ be a model $G \times H$ singularity of codimension $s$. Assume furthermore that the manifold collection $K$ carries a fundamental class and satisfies the hypotheses of Lemma 2.4. Let $K(X, Y)$ be the $G \times H$ singularity defined by the given $G$-structure on $E, H$-structure on $E^{\prime}$, and the model $G \times H$ singularity $K$.

(3) Let $f: \xi \rightarrow \eta$ be a homomorphism of smooth vector bundles covering the smooth map $\bar{f}: X \rightarrow Y$. Assume that $f$ displays the singularity $K$ transversally.

Then $S_{K}(f)$ the manifold collection of $K$-singular points of $f$ carries a fundamental class $\left[S_{K}(f)\right]$. Let $i: S_{K}(f) \rightarrow X$ be the inclusion map and let $D_{X}$ be Poincaré duality in $X$. Then there exists a polynomial $P$, which is independent of $f$, and universally defined, such that

$$
D_{X} i_{*}\left[S_{K}(f)\right]=P\left(W_{i}^{G}(\xi), \bar{f}^{*} W_{j}^{H}(\eta)\right)
$$

THEOREM 2.6. Let us assume the hypotheses of 2.5 except the requirement that $G_{c}=O(k)$ and $H_{c}=O(t)$. We still have the fact that $S_{K}(f)$ carries a fundamental class $\left[S_{K}(f)\right]$. However the polynomial condition is replaced by the following condition. There exists $Z_{2}$-characteristic classes $a_{i} \in H^{i}(X)$ of the $G$-structure, and $Z_{2}$-characteristic classes $b_{i} \in H^{k-i}(Y)$ of the $H$-structure, such that

$$
D_{X} i_{*}\left[S_{K}(f)\right]=\sum_{i=0}^{k} a_{i} \cup \bar{f}^{*} b_{i}
$$

Furthermore the $a_{i}$ and $b_{i}$ are pull backs by the classifying maps of universally defined classes $\alpha_{i}$ and $\beta_{i}$.

Proof. We will prove these theorems simultaneously. Let the dimension of $X$ be $n$ and the dimension of $Y$ be $N$. We know that $B_{G}^{n+N+1} \times B_{H}^{n+N+1}$ is an $(n+N+1)-$ universal classifying space for principal $G \times H$-bundles. Let $h \in\left[X \times Y, B_{G_{c} \times H_{c}}^{n+N+1}\right]$ be the classifying map for the principal $G \times H$ bundle given by the $G$-structure on $E$, and the $H$-structure on $E^{\prime}$. Let $\tilde{E}$ be the universal vector bundle of fiber Hom $\left(R^{p}, R^{q}\right)$ over $B_{G}^{n+N+1} \times B_{H}^{n+N+1}$ given by the representations $\varphi_{1}$ and $\varphi_{2}$. We know that $h^{-1}(\widetilde{E})=\operatorname{Hom}\left(E, E^{\prime}\right)$. Let $\psi$ be the corresponding bundle map which sends the fiber over $(x, y)$ isomorphically onto the fiber over $h(x, y)$. Then 


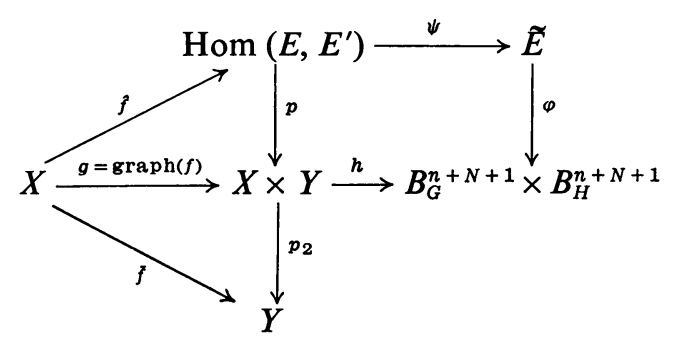

Diagram 1

consider the above commutative diagram where $p$ and $\varphi$ are bundle projections, and $p_{2}$ is the projection onto the second factor.

Let us consider the commutative diagram (2), where $p_{1}, p_{2}, \bar{p}_{1}$ and $\bar{p}_{2}$ are just projections onto the factors of a product, and $\pi_{1}$ and $\pi_{2}$ are the classifying maps for the principal $G$-bundle $P_{G}$ and the principal $H$-bundle $P_{H}$ given by the $G$ structure on $E$ and the $H$-structure on $E^{\prime}$ respectively. Let $\zeta \in H^{k}\left(B_{G}^{n+N+1} \times B_{H}^{n+N+1}\right)$.

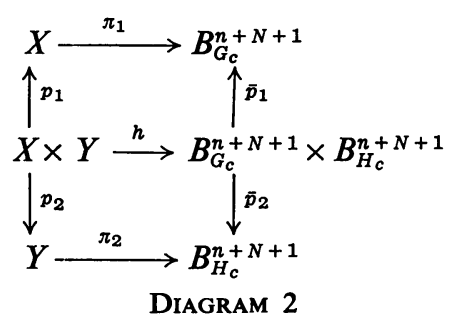

Then by the Künneth theorem and the definition of the cup product, there exist classes $\alpha_{i} \in H^{i}\left(B_{G_{c}}^{n+N+1}\right)$ and $\beta_{i} \in H^{k-i}\left(B_{H_{c}}^{n+N+1}\right)$ such that

$$
\zeta=\sum_{i=0}^{k} \bar{p}_{1}^{*}\left(\alpha_{i}\right) \cup \bar{p}_{2}^{*}\left(\beta_{i}\right) .
$$

If we are in the situation of 2.5 , Theorems 1.12 imply that each $\alpha_{i}$ and $\beta_{i}$ can be expressed uniquely as polynomials in the classes $W_{1} \cdots W_{k}$ which generate $H^{*}\left(B_{O(k)}^{n+N+1}\right)$ and the classes $\tilde{W}_{1} \cdots \tilde{W}_{t}$ which generate $H^{*}\left(B_{O(t)}^{n+N+1}\right)$ respectively. Hence there exists a unique polynomial $P$ such that

$$
\zeta=P\left(\bar{p}_{1}^{*} W_{i} ; \bar{p}_{2}^{*} \tilde{W}_{j}\right) .
$$

Then

$$
\begin{aligned}
h^{*} \xi & =P\left(h^{*} \bar{p}_{1}^{*} W_{i}, h^{*} \bar{p}_{2}^{*} \tilde{W}_{j}\right) \\
& =P\left(p_{1}^{*} \pi_{1}^{*} W_{i}, p_{2}^{*} \pi_{2}^{*} \tilde{W}_{j}\right) \\
& =P\left(p_{1}^{*} W_{i}^{G}(\xi), p_{2}^{*} W_{j}^{H}(\eta)\right), \quad \text { in the case of } 2.5
\end{aligned}
$$


In the case of 2.6 we have $h^{*} \xi=\sum_{i=0}^{k} p_{1}^{*} \pi_{1}^{*}\left(\alpha_{i}\right) \cup p_{2}^{*} \pi_{2}^{*}\left(\beta_{i}\right)$. Let $K_{\tilde{E}}$ be the universal $G \times H$ singularity induced by $K$, and let $K(X, Y)$ be the $G \times H$ singularity induced by $K$, and the $G$ and $H$ structures on $E$ and $E^{\prime}$ respectively. The map $\psi$ from diagram (1) is a bundle map, and hence $\psi\left(\operatorname{Hom}\left(E, E^{\prime}\right)\right) \hbar K_{\tilde{E}}$. Hence $K(X, Y)=\psi^{-1}\left(K_{\tilde{E}}\right)$. We know that $K$ carries a fundamental class. Hence by Lemma 2.3 so does $K_{\tilde{E}}$. Let $i^{\prime}: K(X, Y) \rightarrow \operatorname{Hom}\left(E, E^{\prime}\right)$ and $j^{\prime}: K_{\tilde{E}} \rightarrow \widetilde{E}$ be the inclusion maps. Let $D_{\tilde{E}}$ and $D_{\text {Hom }\left(E, E^{\prime}\right)}$ be Poincaré duality in $\widetilde{E}$ and Hom $\left(E, E^{\prime}\right)$ respectively. Hence 2.4 implies that $K(X, Y)$ carries a unique fundamental class $[K(X, Y)]$ such that

$$
\psi^{*} D_{\tilde{E}} j_{*}^{\prime}\left[K_{\tilde{E}}\right]=D_{\text {Hоm }\left(E, E^{\prime}\right)} i_{*}^{\prime}[K(X, Y)] .
$$

We also know that $\hat{f}: X \rightarrow \operatorname{Hom}\left(E, E^{\prime}\right)$ is transverse to $K(X, Y)$. Hence by applying 2.4 again we get $S_{K}(f)$ carries a unique fundamental class $\left[S_{K}(f)\right]$ which furthermore satisfies the relationship

$$
D_{x} i_{*}\left[S_{K}(f)\right]=\hat{f}^{*} D_{\text {Hom }\left(E, E^{\prime}\right)} i_{*}^{\prime}[K(X, Y)] .
$$

The fiber of $\widetilde{E}$ is contractible, hence $\varphi^{*}$ is an isomorphism. Hence there exists a unique class $\xi \in H^{k}\left(B_{G_{c}}^{n+N+1} \times B_{H_{c}}^{n+N+1}\right)$ such that

$$
\varphi^{*}(\xi)=D_{E} j_{*}^{\prime}\left[K_{\tilde{E}}\right] .
$$

Therefore by using the commutativity of the first diagram we have the following result.

$$
\begin{aligned}
D_{x} i_{*}\left[S_{K}(f)\right] & =\hat{f}^{*} D_{\text {Ноm }\left(E, E^{\prime}\right)} i_{*}^{\prime}[K(X, Y)] \\
& =\hat{f}^{*} \psi^{*} D_{\tilde{E} \tilde{E}}^{\prime} j_{*}^{\prime}\left[K_{\tilde{E}}\right] \\
& =\hat{f}^{*} \psi^{*} \varphi^{*}(\xi) \\
& =f^{*} p^{*} h^{*} \xi .
\end{aligned}
$$

We have shown in the foregoing paragraphs that there exists a polynomial $P$ or classes $\alpha_{i} \in H^{i}\left(B_{G_{c}}^{n+N+1}\right)$ and $\beta_{i} \in H^{k-i}\left(B_{H_{c}}^{n+N+1}\right)$ such that

$$
\begin{aligned}
& h^{*} \xi=P\left(p_{1}^{*} W_{1}^{G}(\xi), p_{2}^{*} W_{j}^{H}(\eta)\right) \quad \text { or } \\
& h^{*} \xi=\sum_{i=0}^{k} p_{1}^{*} \pi_{1}^{*}\left(\alpha_{i}\right) \cup p_{2}^{*} \pi_{2}^{*}\left(\beta_{i}\right)
\end{aligned}
$$

depending on whether we are in the situation of 2.5 or 2.6 respectively. Hence in the case of 2.5 we have

$$
\begin{aligned}
D_{X} i_{*}\left[S_{K}(f)\right] & =\hat{f}^{*} p^{*}\left(P\left(p_{1}^{*} W_{i}^{G}(\xi), p_{2}^{*} W_{j}^{H}(\eta)\right)\right) \\
& =g^{*}\left(P\left(p_{1}^{*} W_{i}^{G}(\xi), p_{2}^{*} W_{j}^{H}(\eta)\right)\right) \\
& =P\left(W_{i}^{G}(\xi), \bar{f}^{*} W_{j}^{H}(\eta)\right)
\end{aligned}
$$


In the case of 2.6 we have

$$
\begin{aligned}
D_{X} i_{*}\left[S_{K}(f)\right] & =\hat{f}^{*} p^{*}\left(\sum_{i=0}^{k} p_{1}^{*} \pi_{1}^{*}\left(\alpha_{i}\right) \cup p_{2}^{*} \pi_{2}^{*}\left(\beta_{i}\right)\right) \\
& =g^{*}\left(\sum_{i=0}^{k} p_{1}^{*} \pi_{1}^{*}\left(\alpha_{i}\right) \cup p_{2}^{*} \pi_{2}^{*}\left(\beta_{i}\right)\right) \\
& =\sum_{i=0}^{k} \pi_{1}^{*}\left(\alpha_{i}\right) \cup \bar{f}^{*} \pi_{2}^{*}\left(\beta_{i}\right) .
\end{aligned}
$$

Hence if we let $a_{i}=\pi_{1}^{*}\left(\alpha_{i}\right)$ and $b_{i}=\pi_{2}^{*}\left(\beta_{i}\right)$ we have proven our theorems.

Corollary 2.7. $D_{X} i_{*}\left[S_{K}(f)\right]$ depends only on the homotopy class of $f$, in either the case of 2.5 or 2.6. If the bundle $\eta$ is a trivial bundle, that is if the bundle $P_{H}$ given by the H-structure is trivial, then $D_{X} i_{*}\left[S_{K}(f)\right]$ is independent of $\bar{f}$ all together.

Corollary 2.8. If $G=G L(p, R), H=G L(q, R), \varphi_{1}=i d_{G}$ and $\varphi_{2}=i d_{H}$, then 2.5 reduces to Haefliger's form of the Thom polynomial theorem.

3. Some remarks in cases of geometric interest. In this section we will describe some specific $G$-structures on some vector bundles of geometric interest. We will furthermore comment upon the hypotheses in the polynomial theorem.

Let $X$ be a smooth $n$-dimensional manifold. Let $T_{p}(X)$ denote its $p$ th order tangent bundle, (see [4, §1] or [5, Chapter II]). As we remarked in [4], $T_{p}(X) \cong$ $\left(J_{O}^{p}(X)\right)^{*}$ where $J_{O}^{p}(X)$ denotes the bundle of $p$-jets whose fiber $J_{O}^{p}(X)_{x}$ is the set of $p$-jets of maps from $X$ to $R$ with source $x$ and target $O$. Let $J^{p}(n)$ be the Lie group of invertible $p$-jets from $R^{n}$ to $R^{n}$ with source and target the origin (see [6, §1]). The chain rule defines a natural action of $J^{p}(n)$ on fiber $R^{v(n, p)}$ of $J_{O}^{p}(X)$, and therefore a faithful representation of $J^{p}(n)$ in $G L(\nu(n, p), R)$. This clearly enables us to endow $T_{p}(X)$ with a $J^{p}(n)$ structure in a manner which depends only on the differentiable structure on $X$. This structure will be called the canonical $J^{p}(n)$ structure on $T_{p}(X)$.

Definition 3.1. Let us preserve the notation of 2.5. Assume that $\operatorname{dim} X=n$ and $\operatorname{dim} Y=N$. Let $\left(D^{(k)}\right) k=1,2, \ldots$ be a sequence of $k$ th order dissections on $Y$. Let us assume that $\xi=T_{p}(X)$ and that $\eta=T(Y)$. Let $G=J^{p}(n)$ where the $J^{p}(n)$ structure on $T_{p}(X)$ is the canonical one. Let there be given some arbitrary $H$ structure on $T_{1}(Y)$. Let $K$ be an $J^{p}(n) \times H$ model singularity, and $K(X, Y)$ the corresponding $J^{p}(n) \times H$ singularity, with respect to the $J^{p}(n)$ structure on $T_{p}(X)$, the $H$-structure on $T_{1}(Y)$, and the model singularity $K$. Let $f: X \rightarrow Y$ be a smooth map. Then the vector bundle homomorphism that we want to study will be the $p$ th order osculating map $D_{p} T_{p}(f)$.

(a) Let $H=G L(N)$ and let $\varphi_{2}$ be $i d_{H}$. Then $K(X, Y)$ is called a $p$ th order affine singularity (with respect to the dissections $\left(D^{(k)}\right)$ ). If $D_{p} T_{p}(f)^{\wedge}(x) \in K(X, Y)$ we say 
$f$ has a $p$ th order affine singularity at $x$. If we furthermore assume that the $\left(D^{(k)}, k=1,2, \ldots\right)$ are generated by a single symmetric connection $D^{(1)}$, we can call $K(X, Y)$ a pth order affine singularity (with respect to $D^{(1)}$ ).

(b) Let $H=O(N)$. Let the $H$-structure on $T_{1}(Y)$ be given by a Riemannian structure whose Riemann connection is $D$. If we assume that the sequence $\left(D^{(k)}, k=1,2, \ldots\right)$ is so chosen that $D=D^{(1)}$ then the singularity $K(X, Y)$ is called a $p$ th order Riemannian singularity (with respect to the $\left(D^{(k)}\right)$ ). If the $\left(D^{(k)}\right)$ are all generated by $\nabla$, then the singularity is called a pth order Riemannian singularity.

(c) Let $Y=R^{n}$, and let $D=$ the Riemann connection given by the usual inner product on $R^{n}$. Then let the $O(N)$ structure on $T_{1}\left(R^{n}\right)$ be given by this Riemann structure. Let the $\left(D^{(k)}\right)$ be generated by $D$. In this case the singularity $K(X, Y)$ is called a $p$ th order Euclidean singularity.

Finally we will show that condition (3) of Theorem 2.5 is not very restrictive in any of the cases defined in 3.1 .

Proposition 3.2. Let $X$ and $Y$ be smooth manifolds of dimension $n$ and $N$ respectively. Let $\left(D^{(k)}, k=1,2, \ldots\right)$ be a sequence of $k$ th order dissections on $Y$. Let there be given an $H$-structure on $T_{1}(Y)$ and a $G$-structure on $T_{p}(X)$. Let $K$ be any model $G \times H$ singularity, and let $K(X, Y)$ be the corresponding singularity induced by the G-structure, the H-structure and $K$. Then the set of $f \in C(X, Y)$ such that $D_{p} T_{p}(f)^{\wedge}(X)$ お $K(X, Y)$ is dense in $C(X, Y)$.

Proof. As we said before $K(X, Y)=K_{1}(X, Y) \cup \cdots \cup K_{s}(X, Y)$ is a manifold collection of submanifolds of $\operatorname{Hom}\left(T_{p}(X), T(Y)\right)$. Furthermore $K(X, Y)$ is an admissible submanifold collection of $\operatorname{Hom}\left(T_{p}(X), T(Y)\right)$, in the sense of 4.1 of [4]. Moreover $D_{p} T_{p}(f)^{\wedge}$ is just the map denoted by $D(f)$ in $[4, \S 4]$. Hence this proposition follows immediately from the proof of Theorem 4.3 of [4].

ReMarK. One would like the set maps $f \in C(X, Y)$ such that $D_{p} T_{p}(f)^{\wedge}(X) \hbar$ $K(X, Y)$ to be open as well as dense in $C(X, Y)$. However this is not the case for an arbitrary singularity. If the model singularity $K \subseteq \operatorname{Hom}\left(R^{v(n, p)}, R^{N}\right)$ is a real algebraic variety it is probable that $K$ admits a decomposition into a submanifold collection that is well enough behaved, so that the set of maps which display $K(X, Y)$ transversally is open. Whitney has been able to construct such a decomposition for a complex analytic variety $V \subseteq C^{n}$. (See Whitney's article on local properties of analytic varieties to be published soon by Princeton University Press, in the Morse symposium on topology. Also see $\$ 3$ of [4].) It appears that Whitney's result will also be true for $C$-analytic real analytic varieties, and in particular real. algebraic varieties. However as of yet no one has checked this extension of Whitney's work. Furthermore no one knows at present whether these "good" decompositions of varieties are in any way the "natural" ones for singularity theory. We will now state and prove a proposition which will allow us to conclude the open as well as dense result in some cases of interest. 
Proposition 3.3. Let $X$ and $Y$ be smooth manifolds of dimensions $n$ and $N$ respectively. Let $K=K_{1} \cup \cdots \cup K_{S}$ be a regular submanifold collection of $Y$. Let $\mathscr{C}(X, Y)$ be the space of smooth maps from $X$ into $Y$. Let us assume that $\operatorname{dim} X<$ $\operatorname{codim} K_{2}$ (and therefore $\operatorname{dim} X<\operatorname{codim} K_{i}, i \geqq 2$ ). Then the set of $f \in \mathscr{C}(X, Y)$ such that $f(X) \hbar K$ is open in $\mathscr{C}(X, Y)$.

Proof. Let $f \in \mathscr{C}(X, Y)$ and assume $f(X)$ $K$. Then $f(X) \cap K_{2} \cup \cdots \cup K_{S}=\varnothing$. Let $K_{2} \cup \cdots \cup K_{S}=K_{S^{\prime}} . K_{S^{\prime}}$ is a closed subspace of $Y$, and therefore there exists an open neighborhood $U$ of $K_{S^{\prime}}$ such that $f(X) \cap U=\varnothing$. In fact we can choose $U$ so that $f(X) \cap \bar{U}=\varnothing$. Hence we can construct an open neighborhood $W$ of $f \in \mathscr{C}(X, Y)$ so that if $g \in W$ then $g(X) \cap U=\varnothing$. Let us pick an open neighborhood $V$ of $K_{S^{\prime}}$ such that $U \supset \bar{V} \supset V \supset K_{S^{\prime}}$. Let us pick an admissible atlas $\mathfrak{A}$ of $Y$ of the form $\mathfrak{A}=\mathfrak{A}^{\prime} \cup \mathfrak{A}^{\prime \prime}$, where $\mathfrak{A}^{\prime}$ is an admissible atlas for $Y-\bar{V}, \mathfrak{A}^{\prime \prime}$ is an admissible atlas for some open submanifold $\tilde{U}$ of $Y$ such that $U \supset \bar{U} \sim \widetilde{U} \supset \bar{V}$, and where the families $\mathfrak{A}^{\prime}$ and $\mathfrak{Q}^{\prime \prime}$ are disjoint. Let $K_{V}=K \cap Y-\bar{V}=K_{1} \cap Y-\bar{V}$. Hence $K_{V}$ is a closed submanifold of $Y-\bar{V}$. Let $A=\left\{g \in \mathscr{C}(X, Y-\bar{V}) \mid g(X) \hbar K_{V}\right\}$. Therefore $A$ is open as well as dense in $\mathscr{C}(X, Y-\bar{V})$. Now by our choice of atlases and the definition of the function space topology, $W$ is open in $\mathscr{C}(X, Y-\bar{V})$, and furthermore the set $W$ with the relative topology as a subset of $\mathscr{C}(X, Y)$ is homeomorphic to $W$ with the relative topology as a subset $\mathscr{C}(X, Y-\bar{V})$. Hence $A \cap W$ is open in $\mathscr{C}(X, Y)$ and therefore is the desired neighborhood of $f$.

CoRollary 3.4. Let $X$ and $Y$ be smooth manifolds of dimension $n$ and $N$ respectively. Let $\left(D^{(k)}, k=1,2, \ldots\right)$ be a sequence of $k$ th order dissections on $Y$. Let there be given an $H$-structure on $T_{1}(Y)$ and a G-structure on $T_{p}(X)$. Let $K=$ $K_{1} \cup K_{2} \cup \cdots \cup K_{S}$ be a model $G \times H$ singularity, and let $K(X, Y)$ be the corresponding singularity induced by the above structures. Assume that $n<\operatorname{codim} K_{2}$. Then the set of $f \in C(X, Y)$ such that $D_{p} T_{p}(f)^{\wedge}(X) \hbar K(X, Y)$ is open in $\mathscr{C}(X, Y)$.

Proof. This follows immediately from 3.3 and from 4.3 of [4].

Proposition 3.5. Let us assume the hypotheses of 3.3. Let $f \in \mathscr{C}(X, Y)$, and let $x \in X$ be a point such that $D_{p} T_{p}(f)^{\wedge}(x) \in K(X, Y)$ and $D_{p} T_{p}(f)^{\wedge}$ is transverse to $K(X, Y)$ at $D_{p} T_{p}(f)^{\wedge}(x)$. Then for each neighborhood $U$ of $x$, there exists a neighborhood $W_{f}$ of $f$ in $\mathscr{C}(X, Y)$ with the following property. If $g \in W_{f}$ then there exists a point $x^{\prime} \in U$ at which $D_{p} T_{p}(g)^{\wedge}\left(x^{\prime}\right) \in K(X, Y)$ and $D_{p} T_{p}(f)^{\wedge}$ is transverse to $K(X, Y)$ at $x^{\prime}$.

Proof. This follows immediately from 4.3 of [4] and 8.2 of [6].

4. Some formulas for simple singularities. I. Porteous [8] has given an easy calculation (without Schubert cycles) of some Thom polynomials. However his 
formulas rely on some transversality assumptions which are not identical with the usual transversality assumption. The main object of this section is to show that the usual conditions imply Porteous'.

We will first describe the model $G \times H$ singularity in question. We will fix the following choices throughout this entire section. Let $G=G L(n, R), H=G L(p, R)$, $\varphi_{1}=i d_{G}$, and $\varphi_{2}=i d_{H}$. All singularities described will clearly be $G \times H$ singularities with respect to these representations. Hence no further mention of either $G, H$, or the representations will be made.

Let $q=\min (n, p)$. Let us set $S_{a}=\left(\sigma \in \operatorname{Hom}\left(R^{n}, R^{p}\right) \mid \operatorname{rank}(\sigma)=q-a\right)$. The $S_{a}$ have the following well known properties.

Proposition 4.1 [6, p. 5].

(a) Each $S_{a}$ is a regular submanifold of $\operatorname{Hom}\left(R^{n}, R^{p}\right)$ of dimension

$$
n p-(n-q+a)(p-q+a)
$$

(b) $\bar{S}_{a}=\bigcup_{a^{\prime} \geqq a} S_{a}^{\prime}$.

(c) Each $\bar{S}_{a}$ is a real algebraic variety, and each $S_{a}$ is an algebraic partial manifold.

Let $\pi_{E}: E \rightarrow X$ and $\pi_{F}: F \rightarrow Y$ be smooth vector bundles of fiber dimension $n$ and $p$ respectively. $S_{a}$ then induces a singularity $S_{a}(X, Y) \subseteq \operatorname{Hom}(E, F)$. Let $h: E \rightarrow F$ be a smooth vector bundle homomorphism covering the smooth map $f: X \rightarrow Y$. In this situation we will denote $S_{S_{a}}(h)$ by $S_{a}(h)$. Finally we will let $S_{a}(X)$ be the singularity in Hom $\left(E, f^{-1}(F)\right) \mid X$ induced by $S_{a}$. Due to Proposition 1.8 it is clear that all the information about the singular sets of $h$ is contained in the smooth vector bundle homomorphism over $X, h !: E \rightarrow f^{-1}(F)$. Therefore in the light of 1.8 the remainder of this chapter will consider the situation where both $E$ and $F$ are vector bundles over $X$, and all vector bundle homomorphisms are smooth vector bundle homomorphisms over $X$ (i.e., smooth crosssections of $\operatorname{Hom}(E, F) \mid X)$. Let $S(\operatorname{Hom}(E, F) \mid X)=$ the smooth cross-sections of $\operatorname{Hom}(E, F) \mid X$.

Definition 4.2. Let $h \in S(\operatorname{Hom}(E, F) \mid X)$. We say that $h$ is strongly generic if and only if $h(X) \hbar S_{a}(X)$ for all $a$.

REMARK. If $h: E \rightarrow F$ is a smooth vector bundle homomorphism covering a smooth map $f: X \rightarrow Y$, the $h$ ! is strongly generic if and only if $\hat{h}(X) \hbar S_{a}(X, Y)$ for all $a$.

Finally we can prove the following by a direct calculation using local coordinates.

Proposition 4.3. Let $h \in S(\operatorname{Hom}(E, F) \mid X)$. Let $h^{T} \in S(\operatorname{Hom}(F, E) \mid X)$ be the transpose of $h$. Then

(a) $S_{a}(h)=S_{a}\left(h^{T}\right)$, and

(b) $h(X) \pitchfork S_{a}(X)$ if and only if $h^{T}(X) \pitchfork S_{a}(X)$. (The $S_{a}$ in reference to $h$ is 
always the singular manifold of $\operatorname{Hom}(E, F) \mid X$, and the $S_{a}$ in reference to $h^{T}$ is always the singular submanifold of $\operatorname{Hom}(F, E) \mid X$.)

$N . B$. From now on we will restrict ourselves to the study of the case where $p \geqq n$. In the case where $p \leqq n$ we merely study the transpose of the homomorphism in question.

REMARK. In that $n \leqq p, S_{a}(h)$ is the set of points in $X$ where the rank of the kernel of $h$ is $a$.

Let us recall that $E$ and $F$ are smooth vector bundles over $X$, of fiber dimension $n$ and $p$ respectively. Let $h \in S(\operatorname{Hom}(E, F) \mid X)$. We further assume that $n \leqq p$. Let $x \in S_{a}(h)$. There exists exactly one $a$-plane in $E_{x}$ which $h$ maps into zero. Let $G_{a}(E)$ be the fiber bundle over $X$ with fiber $G_{a, n}$ canonically associated to the vector bundle $E$. Let $s_{a}: S_{a}(h) \rightarrow G_{a}(E)$, map the above point $x$ into the $a$-plane which corresponds to the kernel of $h(x)$. Let $K_{a}$ be the vector bundle of fiber dimension $a$, over $G_{a}(E)$ defined as follows. Pick $(x, P) \in G_{a}(E)$ where $x \in X$ and $P$ is an $a$-plane in $E_{x}$. Then $\left(K_{a}\right)_{(x, P)}$ is the $a$-dimensional subspace of $E_{x}$ determined by $P$. Let $\tilde{E}$ be the vector bundle over $G_{a}(E)$ given by the relation $\widetilde{E}_{(x, P)}=E_{x}$ and let $\tilde{F}$ be the bundle over $G_{a}(E)$ given by the relation $\tilde{F}_{(x, P)}=F_{x}$. Let $i$ be the canonical bundle monomorphism of $K_{a}$ into $\tilde{E}$. Let $M_{a}$ be the cokernel of $i$. Let $\tilde{h} \in S\left(\operatorname{Hom}(\tilde{E}, \tilde{F}) \mid G_{a}(E)\right)$ be given by the formula $\tilde{h}(x, P)=h(x)$. Finally set $h^{a}=\tilde{h} i, h^{a} \in S\left(\operatorname{Hom}\left(K_{a}, \tilde{F}\right) \mid G_{a}(E)\right)$. This gives us the following commutative diagram of vector bundles over $G_{a}(E)$. Let $\pi_{a}: G_{a}(E) \rightarrow X$ be the bundle projection.

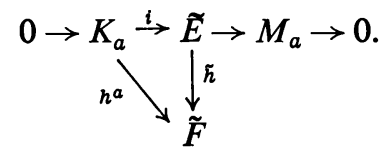

Then it is clear that $h^{a}(x, p)=h(x) \mid K_{a(x, p)}$. Then these definitions yield the following proposition.

Proposition 4.4. (a) $s_{a}\left(S_{a}(h)\right) \subseteq S_{a}\left(h^{a}\right)$.

(b) $\pi_{a} s_{a}=i d_{\mathrm{X}}$.

(c) $\pi_{a}\left(S_{a}\left(h^{a}\right)-s_{a}\left(S_{a}(h)\right)\right)=\bigcup_{a^{\prime}>a} S_{a^{\prime}}(h)$.

DEFINITION 4.5. We say that $h$ is a fully a-transversal to zero, if $h^{a}$ is transversal to the zero section of $\operatorname{Hom}\left(K_{a}, \tilde{F}\right) \mid G_{a}(E)$ at points of $S_{a}\left(h^{a}\right)$.

We will now state Porteous' main result. The proof of this theorem is to be found in [8].

TheOREM 4.6 (Thom-Porteous). Let $\pi: E \rightarrow X$, and $\pi_{1}: F \rightarrow Y$ be smooth vector bundles of fiber dimension $n$ and $p$ respectively. Let $h: E \rightarrow F$ be a smooth vector 
bundle homomorphism over the smooth map $f: X \rightarrow Y$. Let $D_{X}$ be Poincaré duality in $X$. Let $i: S_{a}(h) \rightarrow X$ be the inclusion map. Let $W(E)=1+W_{1}(E) x+\cdots+W_{n}(E) x^{n}$ and let $f^{*} W(F)=1+f^{*} W_{1}(F) x+\cdots+f^{*} W_{p}(E) x^{p}$. Let $W(E)^{-1}$ and $f^{*} W(F)^{-1}$ be the formal inverse to each of the above polynomials respectively. Let $A_{i}=$ the coefficient of $x^{i}$ in the polynomial $f^{*} W(F) \cup W(E)^{-1}$ and let $B_{i}$ be the coefficient of $x^{i}$ in the polynomial $W(E) \cup f^{*} W(F)^{-1}$.

(a) Assume that $n \leqq p$, and that $h !: E \rightarrow f^{-1} F$ is fully a-transversal to zero. Then $\left(S_{a}(h)\right)^{-}$carries a fundamental class $\left[S_{a}(h)\right]^{-}$and $D_{X}\left(i_{*}\left[S_{a}(h)\right]^{-}\right)$is the determinant of the a $\times$ a matrix with $(i, j)$ th entry $A_{p-n+a-i+j} i, j=1, \ldots, a$.

(b) Assume that $n>p$, and that $h !^{T}$ is fully a-transversal to zero. Then $\left(S_{a}(h)\right)^{-}$ carries a fundamental class $\left[S_{a}(h)\right]^{-}$and $D_{X}\left(i_{*}\left[S_{a}(h)\right]^{-}\right)$is the determinant of the $a \times a$ matrix with $(i, j)$ th entry $B_{n-p+a-i+j}, i, j=1, \ldots, a$.

In order to use this theorem we will need the following result.

THEOREM 4.7. Let $h \in S(\operatorname{Hom}(E, F) \mid X)$. If $h$ is strongly generic, then $h$ is fully a-transversal to zero. (We have assumed Fiber $\operatorname{dim}(E) \leqq$ Fiber $\operatorname{dim}(F)$.) We need not assume that $X$ is connected.

Proof. Pick $\left(x_{0} P_{0}\right) \in S_{a}\left(h^{a}\right)$. Hence $x \in S_{a^{\prime}}(h), a^{\prime} \geqq a$, and $P_{0}$ is an $a$-plane such that the $a$-dimensional subspace determined by $P_{0}$ in $E_{x_{0}}$ is contained in the kernel of $h\left(x_{0}\right)$. Let $\operatorname{dim}(X)=k$. Let $U$ be a coordinate neighborhood of $x_{0}$ with coordinate functions $\left(x_{1}, \ldots, x_{k}\right)$. Let us further assume that $U$ is a trivializing neighborhood of any vector bundle we need in this proof. (They will be finite in number so this assumption is harmless.) As before we have a map $\theta h: U \rightarrow$ Hom $\left(R^{n}, R^{p}\right)$. Let us pick bases $\left(e_{1}, \ldots, e_{n}\right)$ of $R^{n}$ and $\left(f_{1}, \ldots, f_{p}\right)$ of $R^{p}$ with the following properties when viewed as bases of $E_{x_{0}}$ and $F_{x_{0}}$ respectively.

(a) $\left(e_{1}, \ldots, e_{a}\right)$ span the plane $P_{0}$ at $x_{0}$.

(b) $h\left(x_{0}\right)\left(e_{i}\right)=0$ for $i=1, \ldots, a^{\prime}$.

(c) $h\left(x_{0}\right)\left(e_{n}\right)=f_{p}, h\left(x_{0}\right)\left(e_{n-1}\right)=f_{p-1}$, and $h\left(x_{0}\right)\left(e_{a^{\prime}+1}\right)=f_{p-n+a^{\prime}+1}$. With these bases we will pick the usual coordinates $f_{i j}$ on $\operatorname{Hom}\left(R^{n}, R^{p}\right)=R^{n p}$. Let $\left(a_{i j}(x)\right)=$ $\left(r_{i j}(\theta h(x))\right)$. Then the bases were picked so that

$$
\begin{gathered}
\left(a_{i j}\left(x_{0}\right)\right)=\underset{c \mid c}{0} \\
\left.\begin{array}{c}
p-n+a^{\prime} \quad n-a^{\prime} \\
I_{n-a^{\prime}}
\end{array}\right)_{n-a^{\prime} .}^{a^{\prime}}
\end{gathered}
$$

That is $a_{i j}\left(x_{0}\right)=0, i=1, \ldots, a^{\prime}, a_{i j}\left(x_{0}\right)=0, i \geqq a^{\prime}, j \leqq p-n-a^{\prime}$ and

$$
a_{a^{\prime}+t, p-n+a^{\prime}+s}\left(x_{0}\right)=\delta_{t, s}
$$


Let us pick neighborhoods $V$ of $x_{0}$, and $\tilde{U}$ of $\theta(h \mid U)\left(x_{0}\right)$ such that $(\theta h \mid U)(V) \subseteq \tilde{U}$. Furthermore assume

$$
\theta(h \mid U)(x)=\left(\frac{D(x) \mid C(x)}{B(x) \mid A(x)}\right)
$$

where $A(x)$ is a nonsingular $n-a^{\prime}$ square matrix for each $x \in V$. Finally define

$$
\Psi(x)=D(x)-C(x) A^{-1}(x) B(x),
$$

and we know by hypothesis that $S_{a^{\prime}} \cap \tilde{U}=\Psi^{-1}(o) . R^{a^{\prime}\left(p-n-a^{\prime}\right)}$ can be given coordinates by looking at it as $\operatorname{Hom}\left(R^{a^{\prime}}, R^{p-n+a^{\prime}}\right)$. Let $S_{i j}$ be such coordinates so that $S_{i j}(\Psi(x))=D(x)_{i j}-\left(C(x) A(x)^{-1} B(x)\right)_{i j}, i=1, \ldots, a^{\prime}, j=1, p-n-a^{\prime}$. Hence by our choice of bases and coordinates $\partial S_{i j}\left(\Psi\left(x_{0}\right)\right) /\left.\partial x_{\alpha}\right|_{x_{0}}=\partial D_{i j}\left(x_{0}\right) /\left.\partial x_{\alpha}\right|_{x_{0}}$. Let us consider $\left(\partial D_{i j}\left|\partial x_{\alpha}\right|_{x_{0}}\right)$ for fixed $(i, j)$ and $\alpha=1, \ldots, k$ as a vector in $R^{k}$. Then by hypothesis the set of $a^{\prime}\left(p-n+a^{\prime}\right)$ such vectors (one for each choice of $\left.(i, j)\right)$ are linearly independent.

Let $U$ be the coordinate neighborhood of $x_{0}$ chosen above. Then $\pi_{E}^{-1}(U)=$ $U \times R^{n}$, and let $\left(e_{1}, \ldots, e_{n}\right)$ be the basis of $R^{n}$, chosen in the last paragraph. Let $P_{0} \in G_{a}(E)_{x_{0}}$ be the $a$-plane in question. Let us consider $R^{n}$ as $n$-tuples of reals with $e_{i}=(0, \ldots, 1, \ldots, 0), 1$ in the $i$ th place. We now construct a neighborhood $V$ of $P_{0}$ as an element of $G_{a}$ such that $U \times V \subset \pi_{a}^{-1}(U)$ will be a coordinate neighborhood of $\left(x_{0}, P_{0}\right)$. We follow [9, p. 46-47].

Let

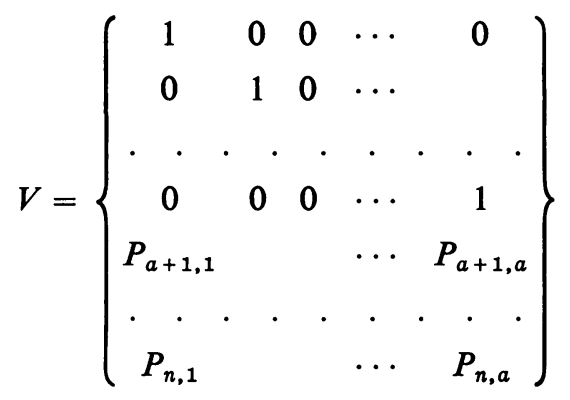

where each matrix represents the $a$-plane spanned by the column vectors which are linearly independent vectors in $R^{n} ; a$ in number. At $P_{0}$ all the $P_{i j}=0$. We will also assume that the $P_{i j}$ are sufficiently small. Hence $U \times V$ is the desired neighborhood of $\left(x_{0}, P_{0}\right)$ with coordinate functions given by the $\left(x_{i}, p_{j k}\right)$. Let $\rho_{a}$ be the bundle projection map of $\operatorname{Hom}\left(K_{a}, \tilde{F}\right) \mid G_{a}(E)$. We further assume that $U \times V$ is a trivializing neighborhood of $\operatorname{Hom}\left(K_{a}, \tilde{F}\right) \mid G_{a}(E)$. Then we have $\rho_{a}^{-1}(U \times V)=$ $U \times V \times \operatorname{Hom}\left(R^{a}, R^{p}\right)$. Let $(x, P) \in U \times V$. We want to put a good set of coordinates on Hom $\left(K_{a}, \tilde{F}\right) \mid G_{a}(E)(x, P) . K_{a}(x, P)$ is a vector space which has as a basis the vectors $\left(e_{i}+\sum_{k=a+1}^{n} p_{k, i} e_{k}\right), i=1, \ldots, a . \tilde{F}(x, P)$ is a vector space with the vectors 
$\left(f_{j}\right), j=1, \ldots, p$ as a basis. We can then use these bases to pick coordinate functions $t_{i j}, i=1, \ldots, a, j=1, \ldots, p$ of $\operatorname{Hom}\left(R^{a}, R^{p}\right)$ at each point $(x, P) \in U \times V$. Let $t_{i j}$ be the $(i, j)$ th entry of the matrix of a linear transformation given by the bases $\left(e_{i}+\sum p_{k, i} e_{k}\right)$ and $\left(f_{j}\right)$. Then the functions $\left(x_{i}, p_{k j}, t_{s r}\right)$ are functionally independent and form a set of coordinate functions on $U \times V \times \operatorname{Hom}\left(R^{a}, R^{p}\right)$.

Assume that $k \geqq a^{\prime}\left(p-n+a^{\prime}\right)$ and therefore $k+a(n-a) \geqq a p$ (otherwise the theorem is trivial). Let $h^{a}: K_{a} \rightarrow \tilde{F}$ be the vector bundle homomorphism over $G_{a}(E)$ induced by $h$. Then we can view $h^{a}$ as an element of $S\left(\operatorname{Hom}\left(K_{a}, \tilde{F}\right) \mid G_{a}(E)\right)$. Then $h^{a}(x, P)\left(e_{i}+\sum_{k=a+1}^{n} p_{k, i} e_{k}\right)=\sum_{j=1}^{p}\left(a_{i j}(x)+\sum_{k=a+1}^{n} p_{k, i} e_{k, j}(x)\right) f_{j}$, where $\left(a_{i j}(x)\right)$ is the matrix defined in the first paragraph of this proof. Hence

$$
t_{i j}\left(h^{a}(x, P)\right)=a_{i j}(x)+\sum_{k=a+1}^{n} p_{k, i} a_{k, j}(x) \text { for } i=1, \ldots, a \text {, and } j=1, \ldots, p \text {. }
$$

We know that $k+a(n-a) \geqq a p$. Let us fix $(i, j)$ then the set

$$
\left(\left.\frac{\partial t_{i j}\left(h^{a}(x, P)\right)}{\partial x_{\alpha}}\right|_{\left(x_{0} P_{0}\right)},\left.\frac{\partial t_{i j}\left(h^{a}(x, P)\right)}{\partial p_{k^{\prime}, j^{\prime}}}\right|_{\left(x_{0} P_{0}\right)}\right)
$$

for each $(\alpha=1, \ldots, k)\left(k^{\prime}=a+1, \ldots, n\right),\left(j^{\prime}=1, \ldots, a\right)$, can be considered as a vector in $R^{k+a(n-a)}$. By 3.3 of [4] we will have demonstrated this theorem if we can show that these $a p$ vectors in $R^{k+a(n-a)}$ space are linearly independent.

$$
\begin{gathered}
\left.\frac{\partial t_{i j}\left(h^{a}(x, P)\right)}{\partial x_{\alpha}}\right|_{\left(x_{0} P_{0}\right)}=\left.\frac{\partial \alpha_{i j}(x)}{\partial x_{\alpha}}\right|_{\left(x_{0} P_{0}\right)}=\left.\frac{\partial D_{i j}}{\partial x_{\alpha}}\right|_{\left(x_{0} P_{0}\right)} \begin{array}{l}
i=1, \ldots, a \\
j=1, \ldots, p-n+a^{\prime},
\end{array} \\
\left.\frac{\partial t_{i j}\left(h^{a}(x, P)\right)}{\partial P_{s, t}}\right|_{\left(x_{0}, P_{0}\right)}=0 \text { for } \begin{array}{l}
i=1, \ldots, a \\
j=1, \ldots, p-n+a^{\prime} .
\end{array}
\end{gathered}
$$

Hence these are linearly independent. Let us look at

$$
\begin{aligned}
& \left.\frac{\partial t_{i j}\left(h^{a}(x, P)\right)}{\partial P_{s, t}}\right|_{\left(x_{0}, P_{0}\right)} \quad \text { for } \begin{array}{l}
i=1, \ldots, a \\
j=p-n+a^{\prime}+1, \ldots, p,
\end{array} \\
& \begin{aligned}
\left.\frac{\partial t_{i j}\left(h^{a}(x, P)\right)}{\partial P_{s, t}}\right|_{\left(x_{0}, P_{0}\right)} & =\sum_{k=a^{\prime}+1}^{n} \frac{\partial P_{k, t}}{\partial p_{s, t}} a_{k, j}\left(x_{0}\right) \\
= & \delta_{i, t} \sum_{k^{\prime}=1}^{n} \delta_{a^{\prime}+k^{\prime}, s} a_{a^{\prime}+k^{\prime}, j}\left(x_{0}\right) .
\end{aligned}
\end{aligned}
$$

But

$$
a\left(x_{0}\right)_{a^{\prime}+k^{\prime}, p-n+a^{\prime}+j^{\prime}}=\delta_{k^{\prime}, j^{\prime}}
$$


Hence

$$
\left.\frac{\partial t_{i j}\left(h^{a}(x, P)\right)}{\partial p_{s, t}}\right|_{\left(x_{0}, P_{0}\right)}=\delta_{i, t} \delta_{j, p-n+s}
$$

This shows that the set of vectors in question is linearly independent.

\section{Part II - The Geometry of Immersions}

We will begin by setting up some notation and conventions. Let $E$ be a smooth vector bundle over $X$. Let $F$ be the ring of smooth real valued functions on $X$. Then $S(E)$ will always denote the $F$-module of smooth sections of $E$. By a linear connection on $E$ will be meant a linear connection on the $F$-module $S(E)$. Then a linear connection on $E$ induces a linear connection on any coordinate neighborhood $V$ of $X$ as follows. Let $u \in \Gamma(V, \mathbf{T}(\mathbf{X}))$ and let $T \in \Gamma(V, \mathbf{S}(\mathbf{E}))$. Then extend $u$ and $T$ to $\tilde{u}$ and $\tilde{T}$ in $S(T(X))$ and $S(E)$ respectively. It is well known that $\left(\nabla_{\tilde{u}} \tilde{T}\right)(x)$ for $x \in V$ is independent of the extensions picked. Hence we have the desired connection. This localization will be used often in what follows without further comment.

In [4] we confused $p$ th order dissections on $X$ with splittings of the short exact sequence

$$
0 \rightarrow T_{p}(X) \rightarrow T_{p+1}(X) \rightarrow O^{p+1} T(X) \rightarrow 0
$$

they induced. No confusion arose from this because we dealt only with the latter concept. In this chapter we will be dealing with both the connections and the splittings on the left. We will denote them both by the same letter say $\nabla$. However an expression of the form $\nabla_{u} T$ will always mean the connection, while one of the form $\nabla(u T)$ will always mean the splitting. In most cases it will be clear from the context which one we are considering.

Finally, let $f: E \rightarrow E^{\prime}$ be a homomorphism of smooth vector bundles over $X$. Then $f$ induces a homomorphism of $F$-modules $S(f): S(E) \rightarrow S\left(E^{\prime}\right)$ in the obvious way. We will suppress the notation " $S(f)$ " in favor of $f$, as it will be obvious when we are dealing with sections and when we are not. Furthermore throughout Part II the manifolds denoted by $X$ and $Y$ need not be connected unless it's explicitly stated to the contrary.

5. Preliminaries and definitions. Let $X$ and $Y$ be smooth manifolds of dimensions $n$ and $N$ respectively. Let $\left(D^{(k)}, k=1,2, \ldots\right)$ be a sequence of $k$ th order dissections in $Y$. Let $f: X \rightarrow Y$ be a $p$ th order of immersion of $X$ in $Y$ and let $N_{f, Y}^{p}(X)$ be the $p$ th order normal bundle of $f$. Let $v_{f}^{p}: O^{p+1} T_{1}(X) \rightarrow N_{f, Y}^{p}(X)$ be the $p$ th order normal form of $f$. In this section we would like to see what relation the map $v_{f}^{1}: O^{2} T_{1}(X) \rightarrow N_{f, Y}^{1}(X)$ bears the more classically defined "second fundamental forms." It is this relation that motivates the generalizations that we make to the " $p$ th order case." In all our applications we will be considering $J^{p}(n) \times H$ 
singularities of the appropriate pth order osculating map. But the "geometric" content and interest in these singularities is that they are singularities in some sense of the second fundamental form. Before we tie these two theories together we will need some lemmas of a technical nature which will facilitate calculations in this and future papers. The following can be found in most texts on differential geometry.

Proposition 5.1. Let $E$ be a smooth vector bundle of fiber dimension $k$ over $X$. Let $\nabla$ be a linear connection on $E$. Let $u \in S\left(T_{1}(X)\right)$ and $T \in S(E)$. Let $x \in X$. Then $\left(\nabla_{u} T\right)(x)$ depends only upon the values of $T$ along the integral curve of $u$, through $x$ in a neighborhood of $x$, provided $u(x) \neq 0$.

Hence we can make the following remarks.

REMARK I. Let $x_{0} \in X$. Let $\gamma$ be a smooth nonsingular curve in a neighborhood $V$ of $x_{0}$ such that $\gamma(0)=x_{0}$. Let $u_{t}=d \gamma(t) / d t$ be the tangent vector to $\gamma$ at $\gamma(t)$. Let $E$ be a smooth vector bundle over $X$, and let $\nabla$ be a linear connection on $E$. Let $T_{t}=T(\gamma(t))$ be a smooth section of $E$ over the curve $\gamma$. Extend $u_{t}$ and $T_{t}$ to crosssections over $V$. Denote the respective extensions by $\tilde{u}$ and $\tilde{T}$. Then $\left(\nabla_{\tilde{u}} \tilde{T}\right)(\gamma(t))$ is independent of the extensions chosen. Hence we could write $\left(\nabla_{\tilde{u}} \tilde{T}\right)(\gamma(t))=$ $\left(\nabla_{u_{t}} T_{t}\right)(\gamma(t))$ unambiguously.

REMARK II. Let $X$ and $Y$ be smooth manifolds of dimension $n$ and $N$ respectively. Let $\nabla$ be a linear connection on $T_{p}(Y)$. Let $f: X \rightarrow Y$ be an immersion. Let $x \in X$ and let $V$ be a coordinate neighborhood of $x$ such that $f \mid V$ is an embedding. Pick $u \in \Gamma\left(V, T_{1}(X)\right)$ and $T \in \Gamma\left(V,\left(f^{-1} T_{p}(Y)\right)\right)$. Then $T_{1}(f) u$ and $T$ can be considered respectively as sections of $T_{1}(Y)$ and $T_{p}(Y)$ over $f(V)$. Assume that $\tilde{V}$ is a coordinate neighborhood of $f\left(x_{0}\right)$ such that $f(V) \subseteq \tilde{V}$. Let $\tilde{u}$ and $\tilde{T}$ be extensions of $T(f) u$ and $T$ to cross-sections over $\tilde{V}$ respectively. Then $\left(\nabla_{\tilde{u}} \tilde{T}\right)\left(f\left(x_{0}\right)\right)$ is independent of the extensions picked. Hence $\left(\nabla_{\tilde{u}} \tilde{T}\right)\left(f\left(x_{0}\right)\right)$ can be unambiguously denoted by $\left(\nabla_{T(f) u} T\right)\left(f\left(x_{0}\right)\right)$. This enables us to define an induced connection on $f^{-1}\left(T_{p}(X)\right)$ which we will also denote by $\nabla$, as follows. Let $u \in S\left(T_{1}(X)\right)$ and let $T \in S\left(f^{-1}\left(T_{p}(Y)\right)\right)$. We then define $\left(\nabla_{u} T\right)(x)=\left(\nabla_{T(f) u} T\right)(f(x))$, for each $x \in X$. This defines the desired smooth section of $f^{-1}\left(T_{p}(Y)\right)$.

Proposition. 5.2. Let $f: X \rightarrow Y$ be an immersion. Let $x_{0} \in X$, and let $V$ be a coordinate neighborhood of $x_{0}$, such that $f \mid V$ is an embedding. Let $u \in \Gamma\left(V,\left(T_{1}(X)\right)_{-}\right.$ and let $T \in \Gamma\left(V, T_{p-1}(X)\right)_{\text {.. Let }} \cdot$ be function (endomorphism) composition. Then $u \cdot T \in \Gamma\left(V, T_{p}(X)\right)$. As before pick a coordinate neighborhood $\tilde{V}$ of $f\left(x_{0}\right)$ such that $f(V) \subseteq \tilde{V}$. Let us extend $T_{1}(f) u$ and $T_{p-1}(f) T$ to elements $\tilde{u}$ and $\tilde{T}$ of $\Gamma(\tilde{V}, T(Y))_{-}$and $\Gamma\left(\tilde{V}, T_{p-1}(Y)\right)_{-}$respectively. Then $\tilde{u} \cdot \tilde{T}\left(f\left(x_{0}\right)\right)$ is equal to $T_{p}(f)_{x_{0}}(u \cdot T)\left(x_{0}\right)$ and is therefore independent of our extensions. This enables us to write $\tilde{u} \cdot \tilde{T}\left(f\left(x_{0}\right)\right)$ as $(T(f) u) \cdot\left(T_{p-1}(f) T\right)$. 
Proof. Let $\operatorname{dim} X=n$ and let $\operatorname{dim} Y=N$. Pick coordinates $\left(x_{1}, \ldots, x_{n}\right)$ on $V$ and $\left(y_{1}, \ldots, y_{N}\right)$ on $\tilde{V}$ such that

$$
y_{i}\left(f\left(x_{1}, \ldots, x_{n}\right)\right)= \begin{cases}x_{i}, & i \leqq n, \\ 0, & i>n .\end{cases}
$$

There exist unique elements $u_{j}$ and $a_{i_{1} \cdots i_{k}} \in \Gamma(V, \underline{F})$ such that

$$
\begin{gathered}
u=\sum_{j=1}^{n} u_{j} \frac{\partial}{\partial x_{j}}, \\
T=\sum_{\substack{1 \leqq i_{1} \leqq \cdots j k i k \\
k \leqq p}} a_{i_{1} \cdots i_{k}} \frac{\partial^{k}}{\partial x_{i_{1}} \cdots \partial x_{i_{k}}} .
\end{gathered}
$$

Then

$$
T_{1}(f) u\left(x_{p}\right)=\sum_{j=1}^{n} u_{j}\left(x_{1}, \ldots, x_{n}\right) \frac{\partial}{\partial y_{j}}
$$

and

$$
T_{p-1}(f) T(x)=\sum_{\substack{1 \leqq i_{1} \leqq i_{k} \leqq n ; \\ k \leqq p-1}} a_{i_{1} \ldots i k}(x) \frac{\partial^{k}}{\partial y_{i_{1}} \cdots \partial y_{i_{k}}} .
$$

Now extend these to respectively a vector field $\tilde{u}$, and a $(p-1)$ st order vector field $\tilde{T}$ on $\tilde{V}$. Hence

$$
\tilde{u}=\sum_{i=1}^{N} u_{i}\left(y_{1}, \ldots, y_{n} ; y_{n-1}, \ldots, y_{N}\right) \frac{\partial}{\partial y_{i}}
$$

and

$$
\tilde{T}=\sum_{\substack{1 \leqq i_{1} \leqq \cdots \vdots i_{k} \leqq N: \\ k \leqq p-1}} \tilde{a}_{i_{1} \cdots i_{k}}\left(y_{1}, \ldots, y_{n} ; y_{n+1}, \ldots, y_{N}\right) \frac{\partial^{k}}{\partial y_{i_{1}} \cdots \partial y_{i_{k}}}
$$

Hence

$$
\begin{aligned}
\tilde{u} \cdot \tilde{T}= & \sum_{\substack{1 \leqq i \leqq N: \\
1 \leqq i_{1} \leq \ldots \leq i_{k} \leqq N ; \\
k \leqq p-1}} \tilde{u}_{i}\left(y_{1}, \ldots, y_{N}\right)\left[\tilde{a}_{i_{1} \cdots i_{k}}\left(y_{1}, \ldots, y_{n}\right) \frac{\partial^{k+1}}{\partial y_{i} \partial y_{i_{1}} \cdots \partial y_{i_{k}}}\right. \\
& \left.+\frac{\partial \tilde{a}_{i_{1}} \cdots i_{k}}{\partial y_{i}} \frac{\partial^{k}}{\partial y_{i_{1}} \cdots \partial y_{i_{k}}}\right] .
\end{aligned}
$$

Therefore

$$
\begin{aligned}
\tilde{u} \cdot \tilde{T}(f(x))= & \sum_{\substack{1 \leqq i \leq n: \\
1 \leqq i_{1}}} u_{i}\left(x_{1}, \ldots, x_{n}\right) \\
& \times\left[a_{\substack{i_{1} \\
k \leqq i_{k}}}\left(x_{1}, \ldots, x_{n}\right) \frac{\partial^{k+1}}{\partial y_{i} \partial y_{i_{1}} \cdots \partial y_{i_{k}}}+\frac{\partial a_{i_{1}} \cdots i_{k}}{\partial y_{i}} \frac{\partial^{k}}{\partial y_{i_{1}} \cdots \partial y_{i_{k}}}\right]
\end{aligned}
$$


This is so because

$$
\begin{gathered}
\tilde{u}_{i}\left(y_{1}, \ldots, y_{n}, 0, \ldots, 0\right)=\left\{\begin{array}{ll}
u_{i}\left(x_{1}, \ldots, x_{n}\right) & i \leqq n \\
0, & i>n
\end{array}\right. \text { and } \\
\tilde{a}_{i_{1} \ldots i_{k}}\left(y_{1}, \ldots, y_{n} ; 0, \ldots, 0\right)= \begin{cases}a_{i_{1} \ldots i_{k}}\left(x_{1}, \ldots, x_{n}\right) & i_{k} \leqq n \\
0, & i_{k}>n,\end{cases}
\end{gathered}
$$

and therefore

$$
\frac{\partial \tilde{a}_{i_{1} \ldots i_{k}}\left(y_{1}, \ldots, y_{n} ; 0, \ldots, 0\right)}{\partial y_{i}}=0 \quad \text { if } i \leqq n, \text { and } i_{k}>n .
$$

Now

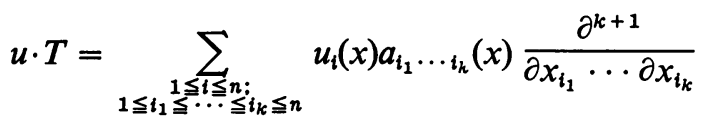

$$
\begin{aligned}
& +\sum_{1 \leqq i_{1} \leqq i \leqq n \leqq i} u_{i}(x) \frac{\partial a_{i_{1}} \cdots i_{k}}{\partial x_{i}}(x) \frac{\partial^{k}}{\partial x_{i_{1}} \cdots \partial x_{i_{k}}} .
\end{aligned}
$$

Hence

$$
\begin{aligned}
& T_{p}(f)(u \cdot T)(x)=\sum_{\substack { 1 \leqq i \leq n \\
1 \leqq i \\
\begin{subarray}{c}{1 \leq i \leq n \\
k \leqq p-1{ 1 \leqq i \leq n \\
1 \leqq i \\
\begin{subarray} { c } { 1 \leq i \leq n \\
k \leqq p - 1 } }\end{subarray}} u_{i}(x) a_{i_{1}} \cdots i_{k}(x) \frac{\partial^{k+1}}{\partial y_{i} \partial y_{i_{1}} \cdots \partial y_{i_{k}}}
\end{aligned}
$$

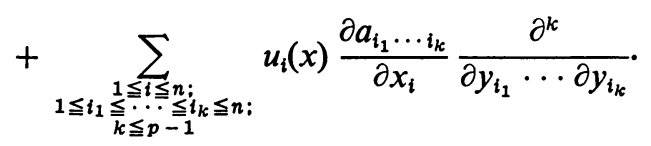

But $x_{i}=y_{i}\left(x_{1}, \ldots, x_{n}\right)$. Hence

$$
\frac{\partial}{\partial x_{i}}\left(a_{i_{1} \ldots i_{k}}\left(y_{1}\left(x_{1}, \ldots, x_{n}\right), \ldots, y_{n}\left(x_{1}, \ldots, x_{n}\right)\right)\right)=\sum_{s} \frac{\partial a_{i_{1}} \cdots i_{k}}{\partial y_{s}} \frac{\partial y_{s}}{\partial x_{i}}=\frac{\partial a_{i_{1}} \cdots i_{k}}{\partial y_{i}} .
$$

Therefore by putting this in the above formula and comparing it with the formula for $\tilde{u} \cdot \tilde{T}(f(x))$ we have our result.

COROLLARY 5.3. Let us assume the hypotheses of 5.2. Let

$$
u_{1}, \ldots, u_{p+1} \in \Gamma\left(V, T_{1}(X)\right)
$$

Then with the same convention

$$
T_{p+1}(f)\left(u_{1} \cdots u_{p+1}\right)=T_{1}(f) u_{1} \cdots T_{1}(f) u_{p+1} .
$$

COROLlaRY 5.4. Let $\nabla^{(p)}$ be a pth order dissection $Y$, and let $\bar{\nabla}^{(p)}$ be the connection $\nabla^{(p)}$ induces on $f^{-1} T_{p}(Y)$. Let $\xi: f^{-1} T_{p}(Y) \rightarrow T_{p}(X)$ be a homomorphism of vector bundles over $X$ such that $\xi T_{p}(f) !=i d$. Then by 9.3 of [4] we have a pth order dissection $\tilde{\nabla}^{(p)}$ on $X$ given by $\xi \bar{\nabla}^{(p)} T_{p+1}(f)$ !. Let $u_{1}, \ldots, u_{p+1} \in S\left(T_{1}(X)\right)$. Then

$$
\tilde{\nabla}^{(p)}\left(u_{1} \cdots u_{p+1}\right)=\tilde{\nabla}^{(p)} u_{1}\left(u_{2} \cdots u_{p+1}\right)=\xi \bar{\nabla}_{u_{1}}^{(p)} T_{p}(f) !\left(u_{2} \cdots u_{p+1}\right) .
$$


Proof. The first equality follows easily from 2.2 of [4]. The second equality follows from 2.2 of [4] and 5.3 and 5.1 of this section.

ReMARK. This tells us that the induced $p$ th order dissection on $X$ is exactly what one would naïvely think it should be. This ends the string of technical lemmas.

Let $X$ and $Y$ be smooth manifolds of dimension $n$ and $N$ respectively. Let $\langle$, be a Riemann metric on $Y$, and let $\nabla^{\prime}$ be the induced Riemann connection on $Y$. Let $f: X \rightarrow Y$ be an immersion and assume $n<N$.

Let $N_{f, Y}(X)$ denote the normal bundle of $f$. Then we have the following commutative diagram of vector bundles over $X$ with exact rows and columns, where the top row is the "pull back" over $X$ of the corresponding sequence over $Y$, and the splitting $\nabla$ is the pull back of the splitting the Riemann connection on $Y$ uniquely determines.

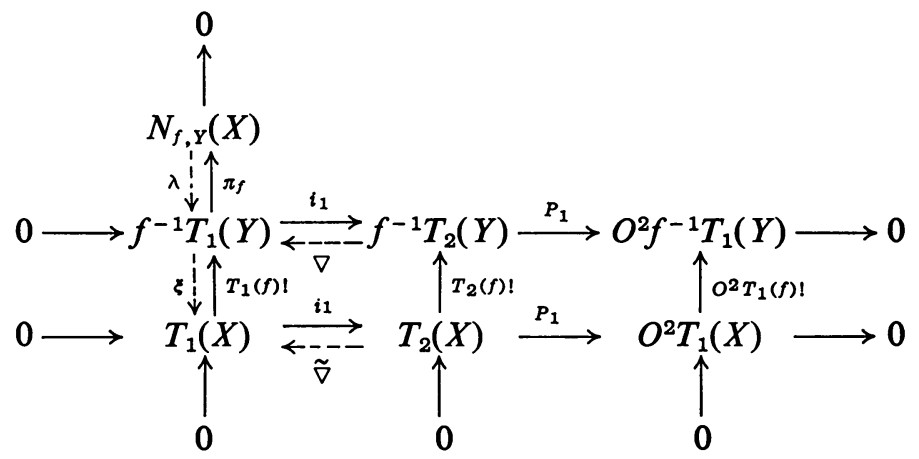

Diagram 5.1

$\pi_{f}$, and $\xi$ are both orthogonal projections given by the induced Riemann structure on $f^{-1} T_{1}(Y) . \lambda$ is the splitting induced by the rest of the data. $\tilde{\nabla}=\xi \nabla T_{2}(f) !$, and is a splitting of the bottom row of Diagram 5.1, and therefore determines uniquely a symmetric linear connection on $X$ denoted by $\tilde{\nabla}^{\prime}$.

Proposition 5.5. $\tilde{\nabla}^{\prime}$ is the Riemann connection of the induced Riemann metric on $X$.

Proof. Let $x \in X$. Let $u, v \in S(T(X))$. As usual we have

$$
\langle u(x), v(x)\rangle_{x}=\left\langle T_{1}(f) u(x), T_{1}(f) v(x)\right\rangle_{f(x)}
$$

for every $x \in X$. Let $\gamma:(-1,1) \rightarrow X$ be a smooth curve. Let $u(t)$ and $v(t)$ stand for $u(\gamma(t))$ and $v(\gamma(t))$ respectively. Let $\tilde{\nabla}_{t}^{\prime} u(t)=\tilde{\nabla}_{d \gamma(t) / d t}^{\prime} u(t)$. Then it suffices to show that

$$
\frac{d}{d t}\langle u(t), v(t)\rangle_{\gamma(t)}=\left\langle\tilde{\nabla}_{t}^{\prime} u(t), v(t)\right\rangle_{\gamma(t)}+\left\langle u(t), \tilde{\nabla}_{t}^{\prime} v(t)\right\rangle_{\gamma(t)} .
$$




$$
\begin{aligned}
\frac{d}{d t}\langle u(t), v(t)\rangle_{\gamma(t)} & =\frac{d}{d t}\left\langle T_{1}(f) u(t), T_{1}(f) v(t)\right\rangle_{f \gamma(t)} \\
& =\left\langle\nabla_{t}^{\prime} T_{1}(f) u(t), T_{1}(f) v(t)\right\rangle_{f \gamma(t)}+\left\langle T_{1}(f) u(t), \nabla_{t}^{\prime} T_{1}(f) v(t)\right\rangle_{j \gamma(t)} \\
& =\left\langle T_{1}(f) \xi \nabla_{t}^{\prime} T_{1}(f) u(t), T_{1}(f) v(t)\right\rangle_{f \gamma(t)} \\
& +\left\langle T_{1}(f) u(t), T_{1}(f) \xi \nabla_{t}^{\prime} T_{1}(f) v(t)\right\rangle_{f \gamma(t)} \\
& =\left\langle T_{1}(f) \tilde{\nabla}\left(\frac{d \gamma(t)}{d t} \circ u(t)\right), T_{1}(f) v(t)\right\rangle \\
& +\left\langle T_{1}(f) u(t), T_{1}(f) \tilde{\nabla}\left(\frac{d \gamma(t)}{d t} \circ v(t)\right)\right\rangle \text { by 5.4, } \\
= & \left\langle T_{1}(f) \tilde{\nabla}_{t}^{\prime} u(t), T_{1}(f) v(t)\right\rangle_{f \gamma(t)}+\left\langle T_{1}(f) u(t), T_{1}(f) \tilde{\nabla}_{t}^{\prime} v(t)\right\rangle_{f \gamma(t)}, \quad \text { by } 2.2 \text { of [4] } \\
= & \left\langle\tilde{\nabla}_{t}^{\prime} u(t), v(t)\right\rangle_{\gamma(t)}+\left\langle u(t), \tilde{\nabla}_{t}^{\prime} v(t)\right\rangle_{\gamma(t) .}
\end{aligned}
$$

Hence our proposition has been demonstrated.

Let us preserve Diagram 5.1. We will however not assume that $Y$ carries a Riemann structure or that $\nabla^{\prime}$ is a Riemannian connection, but merely a symmetric linear connection on $Y$. Let $\xi: f^{-1} T_{1}(Y) \rightarrow T_{1}(X)$ be an arbitrary splitting of the left hand vertical exact sequence of Diagram 5.1. Let $\lambda: N_{f, Y}(X) \rightarrow f^{-1} T_{1}(Y)$ be the splitting on top induced by $\xi$. We will make use of the abuses of notation justified at the end of the last section. Finally let $\nabla^{\prime}$ stand for the connection on $f^{-1} T_{1}(Y)$ induced by $\nabla$ on $Y$ as in 5.1. We will adopt this notation throughout the remainder of this section.

Definition 5.6. Let $u \in S\left(T_{1}(X)\right)$, and let $N \in S\left(N_{f, Y}(X)\right)$. Then $\lambda N \in S\left(f^{-1} T_{1}(Y)\right)$. Let $S_{N} u=-\xi \nabla_{u}^{\prime} \lambda N . S_{N} u \in S\left(T_{1}(X)\right)$, and is called the operator of the second fundamental form of the immersion $f$. Hence for each $N \in N_{f, Y}(X), S_{N} \in \operatorname{End}_{F}\left(S\left(T_{1}(X)\right)\right)$.

Proposition 5.7. Let $u, v \in S\left(T_{1}(X)\right)$. Let $\nabla^{\prime}$ be the linear connection on $f^{-1} T_{1}(Y)$ induced by the symmetric linear connection denoted by $\nabla^{\prime}$ on $Y$. Let $\tilde{\nabla}^{\prime}$ be the connection induced by $\xi \nabla T_{2}(f)$ ! on $X$, where $\nabla$ is the pulled back dissection. Then

$$
\nabla_{u} T_{1}(f) v=T_{1}(f) \tilde{\nabla}_{u}^{\prime} v+\lambda v_{f}^{\prime}(u, v) \quad(\text { Gauss equation })
$$

Proof. $i d_{f^{-1} T_{1}(Y)}=T_{1}(f) \xi+\lambda \pi_{f}$. The reasoning involved in 5.3 yields

$$
\nabla_{T_{1}(f) u}^{\prime} T_{1}(f) v=\nabla\left(T_{2}(f)(u \cdot v)\right) .
$$

Now apply the above formula to this one. Hence

$$
\nabla_{u}^{\prime} T_{1}(f) v=T_{1}(f) \xi \nabla_{T_{1}(f) u}^{\prime} T_{1}(f) v+\lambda \pi_{f} \nabla T_{2}(f)(u \cdot v)=T_{1}(f) \tilde{\nabla}_{u}^{\prime} v+\lambda \pi_{f} T_{2}(f)(u \cdot v) .
$$

Now $P_{1}(u \cdot v)=u \circ v$. Hence $\pi_{f} \nabla T_{2}(f)(u \cdot v)=v_{f}^{\prime}(u, v)$. This proves the proposition. 
REMARK. Hence the normal map measures the difference between the two covariant derivatives.

Corollary 5.8. Let $v \in S\left(f^{-1} T_{1}(Y)\right)$. Then $v=T(f) \xi v+\lambda \pi_{f} v$. Hence

$$
\xi \nabla_{u}^{\prime} v=\tilde{\nabla}_{u}^{\prime} \xi v-S_{\pi_{f v}} u .
$$

Let us assume Diagram 5.1. Let $\tilde{\nabla}^{\prime}$ be the induced Riemann connection on $X$. Definition 5.9. Let $N \in S\left(N_{f, Y}(X)\right)$, and let $u, v \in S\left(T_{1}(X)\right)$. We then define

$$
Q_{N}(u, v)=\left\langle\lambda N, v_{f}^{\prime}(u, v)\right\rangle \text {. }
$$

Hence for each normal vector field we have a smooth quadratic form $Q_{N}$ on the vector fields of $X$. This set of quadratic forms is called the second fundamental form of the immersion $f$.

With the above notation we have the following well-known proposition.

Proposition 5.10. $Q_{N}(u, v)=\left\langle u, S_{N} v\right\rangle=\left\langle\nu_{f}^{\prime}(u, v), \lambda N\right\rangle$.

Proof. $\left\langle T_{1}(f) u, \lambda N\right\rangle=0$.

REMARK. Hence $S_{N}: S\left(T_{1}(X)\right) \rightarrow S\left(T_{1}(X)\right)$ is a selfadjoint operator.

6. Inflection points. We will fix the following notation. Let $X$ and $Y$ be smooth manifolds of dimension $n$ and $N$ respectively. Let $\left(D^{(k)}, k=1,2, \ldots\right)$ be a sequence of $k$ th order dissections on $Y$. Assume that $N \geqq v(n, p)$.

Definition 6.1. Let $f: X \rightarrow Y$ be a $p$ th order nondegenerate immersion of $X$ in $Y$, and let $N_{f, Y}^{p}(X)$ be the $p$ th order normal bundle of $X$ in $Y$ with respect to $f$. Let $\nu_{f}^{p}: O^{p+1} T(X) \rightarrow N_{f, Y}^{p}(X)$ be the $p$ th order normal form of $f . x \in X$ is said to be a pth order inflection point of $f$ if $\nu_{f}^{p}(x)$ is not of maximal rank.

The study of inflection points is the study of the simple (those of \$4) singularities of the map $\nu_{f}^{p}$. This is equivalent by 9.4 of [4] to studying the simple singularities of $D_{p+1} T_{p+1}(f)$ !.

Let $I^{p}(X, Y)$ denote the space of $p$ th order nondegenerate immersions from $X$ to $Y . I^{p}(X, Y)$ is an open subset of $C(X, Y)$ by Theorem 6.2 of [4]. Let

$$
S_{i}\left(\nu_{f}^{p}\right)=\left(x \in X \mid \operatorname{rank}\left(\nu_{f}^{p}(x)\right)=\min (\nu(n, p+1)-\nu(n, p), N-\nu(n, p))-i\right) .
$$

THeOREM 6.2. (a) There exists a dense subset $D \subseteq I^{p}(X, Y)$ such that if $f \in D$, then the set of inflection points of $f$ is the manifold collection $I_{f}=S_{1}\left(\nu_{f}^{p}\right) \cup \cdots \cup S_{k}\left(\nu_{f}^{p}\right)$ of submanifolds of $X$. Furthermore we have the following result.

(b) Let $N \geqq v(n, p+1)+n$ or $\nu(n, p)<N \leqq \nu(n, p+1)-n$. If $f \in D$ then $I_{f}=\varnothing$ and hence $f$ is a $p+1$ order nondegenerate immersion, and furthermore $D$ is open. 
(c) Let $X$ be connected and let $\nu(n, p+1)-n \leqq N<\nu(n, p+1)+n$. Let $f \in D$. It is known that $\left(S_{i}\left(\nu_{f}^{p}\right)\right)^{-}=S_{i}\left(\nu_{f}^{p}\right) \cup S_{i+1}\left(\nu_{f}^{p}\right) \cup \cdots \cup S_{k}\left(\nu_{f}^{p}\right)$. Then $\left(S_{i}\left(\nu_{f}^{p}\right)\right)^{-}$carries a fundamental class $\left[S_{i}\left(\nu_{f}^{p}\right)\right]$. Let $W^{p+1}(X)=1+W_{1}^{p+1}(X) x+\cdots+W_{n}^{p+1}(X) x^{n}$ and let

$$
f^{*} W(Y)=1+f^{*} W_{1}(Y) x+\cdots+f^{*} W_{n}(Y) x^{n} .
$$

Let $W^{p+1}(X)^{-1}$ and $f^{*} W(Y)^{-1}$ be the formal inverse to each of the above polynomials respectively. Let $A_{i}=$ the coefficient of $x^{i}$ in the polynomial $f^{*} W(Y) W^{p+1}(X)^{-1}$ and let $B_{i}=$ the coefficient of $x^{i}$ in the polynomial $W^{p+1}(X) f^{*} W(Y)^{-1}$. Let $D_{X}$ be Poincaré duality in $X$ and let $j: S_{i}\left(\nu_{f}^{p}\right) \rightarrow X$ be the inclusion map.

Assume $N \geqq v(n, p+1)$. Let $\left(A_{N-v(n, p+1)+i-\alpha+\beta}\right)$ be an $i \times i$ matrix with $\alpha$ and $\beta$ $=1 \cdots i$ and with the $(\alpha, \beta)$ entry given by $A_{N-v(n, p+1)+i-\alpha+\beta}$. Then

$$
D_{X} j_{*}\left[S_{i}\left(\nu_{f}^{p}\right)^{-}\right]=\operatorname{det}\left(A_{N-\nu(n, p+1)+i-\alpha+\beta}\right) .
$$

Assume that $N \leqq \nu(n, p+1)$. Let $\left(B_{v(n, p+1)-N+i-\alpha+\beta}\right)$ be an $i \times i$ matrix with $\alpha$ and $\beta=1 \cdots i$ and with the $(\alpha, \beta)$ entry given by $B_{v(n, p+1)-N+i-\alpha+\beta}$. Then

$$
D_{X} j_{*}\left[S_{i}\left(\nu_{f}^{p}\right)^{-}\right]=\operatorname{det}\left(B_{v(n, p+1)-N+1-\alpha+\beta}\right) .
$$

Proof. Part (a) follows immediately from Proposition 9.4 of [4] and Proposition 3.2 of Part I. Part (b) is just a rewording under the influence of 9.4 of [4], of Theorem 6.2 of [4]. Finally Part (c) follows from Part (a) by Theorems 4.6 and 4.7 of Part I.

COROLlaRY 6.3. The exact same result holds with the following modifications of Part (c). Let $A_{i}=$ the coefficient of $x^{i}$ in the polynomial $W\left(O^{p+1} T(X)\right) W\left(N_{f, Y}^{p}(X)\right)^{-1}$, and let $B_{i}=$ the coefficient of $x^{i}$ in the polynomial $W\left(N_{f, Y}^{p}(X)\right) W\left(O^{p+1} T(X)\right)^{-1}$.

Definition 6.4. Let $D$ be the subset of $I^{p}(X, Y)$ of $6.2(\mathrm{a})$. The elements of $D$ will be called inflection generic.

REMARK. Theorem 6.2 would give a pretty complete story about inflection points provided one could calculate the characteristic classes in question. This however hinges on calculating $W\left(O^{p+1} T(X)\right)$ which as we know is computable but not easily so.

Proposition 6.5. Let $S^{1}$ be the circle. Let $Y$ be unorientable. Let $f \in I^{N-1}\left(S^{1}, Y\right)$. Assume that $f^{*} W_{1}(Y) \neq 0$ (i.e., $f$ is unorientable). Then $f$ has an $(N-1)$ st order inflection point.

Proof. Assume that $f$ is inflection generic. Then 6.2 tells us that $D_{X} j_{*}\left[S_{1}\left(\nu_{f}^{N-1}\right)\right]=$ $f^{*} W_{1}(Y) \neq 0$. Hence $f$ has an $(N-1)$ st order inflection point. Assume that $f$ is not inflection generic, that $f^{*} W_{1}(X) \neq 0$, and that $I_{f}=\varnothing$. Then $f$ is an $N$ th order nondegenerate immersion, and hence there exists an open neighborhood $S_{f}$ of $f$ in $I^{N-1}\left(S^{1}, Y\right)$ such that if $g \in S_{f}$ then $g \in I^{N}\left(S^{1}, Y\right)$. Hence by 6.2 there exists an 
element $g \in S_{f}$ such that $f \sim g$ and such that $g$ is inflection generic. However the fact that $g \in I^{N}\left(S^{1}, Y\right)$ implies that $D_{X} j_{*}\left[S_{1}\left(\nu_{g}^{N-1}\right)\right]=0$ as $I_{f}=\varnothing$. But this contradicts our first statement about inflection generic maps.

Corollary 6.6. Let $p=1$. Let $Y$ be an unorientable two dimensional Riemannian manifold. Let $f: S^{1} \rightarrow Y$ be an immersion parameterized by arc length $s$. Then if $f^{*} W_{1}(Y) \neq 0$, there exists a point $t \in S^{1}$ such that $\left(\nabla_{d f / d s} d f / d s\right)(f(t))=0$, where $\nabla$ is the Riemannian connection in $Y$.

We will now give a list of examples of 6.2 in cases where we have made the necessary calculations. Assume throughout the remainder of this section that $p=1$, and that $D=$ the inflection generic maps in question. The calculations appear in $\$ 10$ of [4].

Dimension $X=2$. The cases of interest are $\operatorname{dim}(Y)=4,5$ or 6 .

$$
\begin{aligned}
& \text { If } \operatorname{dim}(Y)=6 \text { and if } f \in D \text { then } D_{X} i_{*}\left[S_{1}\left(v_{f}^{\prime}\right)\right]^{-}=f^{*} W_{2}(Y) . \\
& \text { If } \operatorname{dim}(Y)=5 \text { and if } f \in D \text { then } D_{X} i_{*}\left[S_{1}\left(v_{f}^{\prime}\right)\right]^{-}=f^{*} W_{1}(Y) . \\
& \text { If } \operatorname{dim}(Y)=4 \text { and if } f \in D \text { then } D_{X} i_{*}\left[S_{1}\left(v_{f}^{\prime}\right)\right]^{-}=f^{*} W_{1}(Y)^{2}+f^{*} W_{2}(Y) .
\end{aligned}
$$

(a) Let $f: P_{2}(R) \rightarrow P_{6}(R)$ be the natural embedding. Let $\alpha \in H^{1}\left(P_{2}(R), Z_{2}\right)$ and $\alpha^{\prime} \in H^{1}\left(P_{6}(R), Z_{2}\right)$ be the respective generators of these groups. Then $f^{*} \alpha^{\prime}=\alpha$. Therefore $\alpha^{2}=W_{2}\left(P_{2}(R)\right)=f^{*}\left(\alpha^{\prime}\right)^{2} \neq 0$. Hence we know that any immersion of $P_{2}(R)$ in $P_{6}(R)$ in the same homotopy class as $f$ has inflection points. The exact same fact is true for $P_{2}(R) \subseteq P_{4}(R)$ because $W_{1}\left(P_{4}(R)\right)=$ the generator of $H^{1}\left(P_{4}(R), Z_{2}\right)$, while $W_{2}\left(P_{4}(R)\right)=0$.

(b) Let $f: X \rightarrow G_{2,5}$ be the classifying map for $T(X)$. If the Euler class $\chi(X)$ is odd then all immersions homotopic to $f$ have inflection points.

Dimension $X=4$.

(a) If $\operatorname{dim} Y=17$ and if $f \in D$, then $D_{X} i_{*}\left[S_{1}\left(\nu_{f}^{\prime}\right)\right]^{-}=W_{2}(X)^{2}+f^{*} W_{4}(Y)$. Therefore if $W_{4}(Y)=0$ and if $W_{2}(X)^{2} \neq 0$ we know that $X$ cannot be immersed in $Y$ without inflection points. If $Y=R^{17}$ and $X=P_{2}(C)$ this is just 10.3b of [4].

(b) Let $\operatorname{dim} Y=14$ and assume that $f \in D$. Then

$$
D_{X} i_{*}\left[S_{2}\left(\nu_{f}^{\prime}\right)\right]^{-}=f^{*} W_{1}(Y) W_{3}(X)+f^{*}\left(W_{1}(Y) W_{3}(Y)\right)+W_{2}(X)^{2}+f^{*} W_{2}(Y)^{2} .
$$

Let $X=P_{2}(C)$. Let $Y=$ a manifold such that $W(Y)=1$. ( $R^{14}$ is such a manifold.) Let $\alpha \in H^{2}\left(P_{2}(C), Z_{2}\right)$ be the generator. Then $D_{X} i_{*}\left[S_{2}\left(v_{f}^{\prime}\right)\right]^{-}=\alpha^{2} \neq 0$. Hence for each $f \in I\left(P_{2}(C), Y\right)$ there exists a point $x \in P_{2}(C)$ such that $\operatorname{dim}\left(\operatorname{ker}\left(\nu_{f}^{\prime}(x)\right)\right)=2$.

Finally we ask under what conditions cannot we nondegenerately immerse the $n$-sphere $S^{n}$ in an $N$ dimensional manifold $Y$. Let $N=\nu(n, 2)+n-1$ or $N=$ $v(n, 2)-n+1$. Let $f \in I\left(S^{n}, Y\right)$. Then $D_{X} i_{*}\left[S_{1}\left(\nu_{f}^{\prime}\right)\right]^{-}=f^{*} W_{n}(Y)$, if $f$ is inflection generic. Hence if $f^{*} W_{n}(Y)$ generates $H^{n}\left(S^{n}, Z_{2}\right)$ then $f$ has an inflection point.

REMARK. It is clear that many more examples of this nature exist for $p=1$ and higher $p$ as well. 
7. Totally geodesic points. We will fix the following notation. Let $X$ and $Y$ be smooth manifolds of dimension $n$ and $N$ respectively. Let $\nabla_{k=1,2, \ldots}^{(k)}$ be a sequence of $k$ th order dissections on $Y$.

Definition 7.1. Let $N \geqq \nu(n, p)$, and assume that $f: X \rightarrow Y$ is a $p$ th order nondegenerate immersion of $X$ in $Y$. Let $N_{f, Y}^{p}(X)$ be the $p$ th order normal bundle of $X$ in $Y$ with respect to $f$, and let $\nu_{f}^{p}: O^{p+1} T(X) \rightarrow N_{f, Y}^{p}(X)$ be the $p$ th order normal form. $x \in X$ is said to be a $p$ th order totally geodesic point (with respect to the $\left(\nabla^{(k)}\right)$ ) of the immersion $f$ if $\nu_{f}^{p}(x) \equiv 0$. If $\nu_{f}^{p}(x) \equiv 0$ for all $x \in X, f$ is said to be a $p$ th order totally geodesic immersion. Finally if $f$ is also an embedding $f(X)$ is called a pth order totally geodesic submanifold of $Y$.

REMARK. Totally geodesic immersions are on the opposite end of the spectrum from the nondegenerate immersions, because in the latter immersions $\nu_{f}^{p}(x)$ has as small a kernel as possible.

Definition 7.2. Let $\nabla^{\prime}$ be a symmetric linear connection on $X$. Let $x \in X$, and let $\gamma:(-1,1) \rightarrow X$ be a smooth curve such that $\gamma(0)=x$. Let $u(t)$ be the tangent vector to $\gamma \cdot \gamma$ is said to be geodesic at $x$ if $\left(\nabla_{u(t)}^{\prime} u(t)\right)(x)=0$.

We will now show that the concept in 6.1 corresponds to the usual notion of a totally geodesic immersion in the case where $p=1$. Let $f: X \rightarrow Y$ be an immersion. Let us recall the following diagram of vector bundles over $X$. Let $\zeta$ be a splitting of the left hand sequence of vector bundles. Let $\nabla$ be the dissection induced by $\nabla^{\prime}$, and let $\tilde{\nabla}$ be the induced dissection.

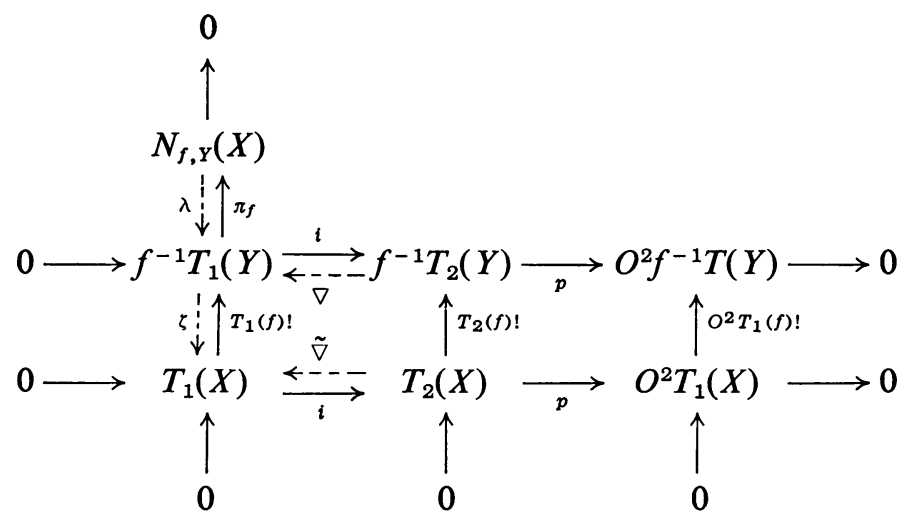

Diagram 7.1

Proposition 7.3. Let $\tilde{\nabla}=\zeta \nabla T_{2}(f)$ be the induced dissection on $X$. Let $x \in X$. $\nu_{f}(x) \equiv 0$ if and only if $f$ maps curves through $x$ which are geodesic at $x$ into curves through $f(x)$ which are geodesic at $f(x)$.

Proof. Let $\gamma(t)$ be any curve on $X$ which is geodesic at $x$. Assume that $\gamma(0)=x$. Let $u(t)$ be the tangent vector to $\gamma(t)$. Then $T_{1}(f)_{\gamma(i)} u(t)$ is the tangent vector to 
$f(\gamma(t))$. We want to show that $\left(\nabla_{T_{1}(f) u(t)}^{\prime} T_{1}(f) u(t)\right)(f(x))=0$, if $\nu_{f}(x)=0$. Proposition 5.7 implies $\nabla_{T_{1}(f) u(t)}^{\prime} T_{1}(f) u(t)=T_{1}(f) \tilde{\nabla}_{u(t)} u(t)+\lambda v_{f}(u(t), u(t))$, where $\tilde{\nabla}^{\prime}$ is the connection induced by $\tilde{\nabla}$. Hence $\left(\nabla_{T_{1}(f) u(t)}^{\prime} T_{1}(f) u(t)\right)(f(x))=0$.

Conversely, assume that $\gamma(t)$ is geodesic at $x$, and furthermore assume $f \gamma(t)$ is geodesic at $f(x)$. Let $u(t)$ be the tangent vector to $\gamma(t)$. Hence

$$
\left(\tilde{\nabla}_{u(t)}^{\prime} u(t)\right)(x)=\left(\nabla_{T_{1}(f) u(t)}^{\prime} T_{1}(f) u(t)\right)(f(x))=0
$$

Hence the above formula tells us that $v_{f}(u(\gamma(0))), u(\gamma(0))=0$ because $\lambda$ is a monomorphism. It is well known that for every $v \in T(X)_{x}$ there exists a geodesic $\eta$ through $x$, such that the tangent vector to $\eta$ at $x$ is $v$. Hence the above calculation implies $v_{f}(v, v)=0$ for all $v \in T(X)_{x}$. Pick $u, v \in T(X)_{x}$.

$$
\nu_{f}(u+v, u+v)=\nu_{f}(u, u)+v_{f}(v, v)+2 v_{f}(u, v)=0
$$

because $v_{f}$ is symmetric. Hence $\nu_{f}(u, v)=0$ at $x$. Therefore $\nu_{f}(x) \equiv 0$.

We will now prove a proposition to indicate now rare it is to have totally geodesic immersions.

Proposition 7.4. Let $N>v(n, p)$, and let $\operatorname{dim} X \geqq 2$. Let $I^{p}(X, Y)$ be the set of pth order nondegenerate immersions of $X$ in $Y$. Then the set of $f \in I^{p}(X, Y)$ without pth order geodesic points is open and dense in $I^{p}(X, Y)$.

Proof. Let $\tilde{q}=\min (N-v(n, p), v(n, p-1)-v(n, p))$. The set of geodesic points of $f$ is clearly the set $S_{\tilde{q}}\left(\nu_{f}^{p}\right)=\left(x \in X \mid \operatorname{rank} \nu_{f}^{p}(x)=0\right)$. Hence by Proposition 9.4 of [4], the set of interest is $S_{\tilde{q}}\left(\nabla_{p+1} T_{p+1}(f)\right.$ !). Hence it will suffice to look at the model singularity $S_{\tilde{q}} \subseteq \operatorname{Hom}\left(R^{v(n, p+1)}, R^{N}\right)$. Let $q=\min (N, v(n, p+1))$. As is well known the codimension in $R^{N \cdot v(n, p+1)}$ of $S_{\tilde{q}}$ is equal to

$$
\begin{aligned}
& (N-q+\tilde{q})(\nu(n, p+1)-q+\tilde{q}) \\
= & (N-v(n, p))(\nu(n, p-1)+\nu(n, p)) \\
= & (N-\nu(n, p))\left(\begin{array}{l}
n+p \\
p+1
\end{array}\right) .
\end{aligned}
$$

Hence the codimension of $S_{\tilde{q}}$ is smallest when $p=1$, and $N-\nu(n, p)=1$. Therefore the codimension of $S_{\tilde{q}}$ in this case is $n(n+1) / 2$. The proposition then follows from Proposition 3.2 and 3.3.

REMARK. This proposition is not true in the case where the dimension of $X$ equals 1 . Proposition 6.5 provides us with a counterexample.

It is well known that for a given Riemann submanifold $X$ of a Riemannian manifold $Y$ to be totally geodesic is very uncommon. The instances are so rare 
one wonders whether there exist manifolds $X \subseteq Y$ so that $X$ is totally geodesic in $Y$ for no Riemann metric on $Y$. It is known that this is not the case for affinely connected manifolds. (See [13, p. 27].) We will now show this is not the case in the Riemannian situation. In fact we will show that there exists a metric on $Y$ such that not only is $X$ totally geodesic but a set of linearly independent cross-sections of the normal bundle of $X$ in $Y$ can be made to be orthonormal.

Proposition 7.5. Let $i: X \rightarrow Y$ be an embedding of a manifold $X$ in a manifold $Y$. Endow $X$ with a Riemannian metric $g_{X}$. Then there exists a Riemannian metric $g_{Y}$ on $Y$ so that $i: X \rightarrow Y$ is an isometric embedding.

Proof. Let $\pi: R(Y) \rightarrow Y$ be the fiber bundle of Riemann metrics on $Y$. Let $p: T_{1}(X) \rightarrow X$ be the tangent bundle of $X$, and let $q: T_{1}(Y) \rightarrow Y$ be the tangent bundle of $Y$. Let $i * T_{1}(Y)$ be the "pull back" of $T_{1}(Y)$ over $X$, and let $N_{i, Y}(X)$ be the normal bundle of the embedding $i$. Hence we have the following short exact sequence of vector bundles over $X$.

$$
0 \longrightarrow T_{1}(X) \stackrel{d i !}{\longleftarrow} i_{t}^{\longrightarrow} T_{1}(Y) \stackrel{j}{\longrightarrow} N_{i, Y}(X) \longrightarrow 0 .
$$

Let $g_{N}$ be a Riemann metric on the fibers of $N_{i, Y}(X)$, and let $t: i^{*} T_{1}(Y) \rightarrow T_{1}(X)$ be a splitting of the above exact sequence. We then define an inner product $g$ on the fibers of $i^{*} T(Y)$ as follows. Let $v, w \in i^{*} T(Y)_{x}$. Then define

$$
g(x)(v, w)=g_{N}(x)(j v, j w)+g_{X}(x)(t v, t w) .
$$

This defines a cross-section of $R(Y)$ over the closed submanifold $i(X)$. Hence by the section extension for fiber bundles we can extend this section to the desired cross-section $g_{Y}$. Let $v, w \in T_{1}(X)_{x}$. Then

$$
\begin{aligned}
g_{Y}(i x)(d i(v), d i(w)) & =g(x)(d i ! v, d i ! w) \\
& =g_{N}(x)(0,0)+g_{X}(x)(t d i ! v, t d i ! w)=g_{X}(x)(v, w)
\end{aligned}
$$

which is what we wanted to show.

Proposition 7.6. Let $\pi: E \rightarrow X$ be a vector bundle. Let $e_{1}, \ldots, e_{s}$ be a set of $s$ linearly independent cross-sections of $E$. Let us consider the following embedding: $\varphi: R^{s} \times X \rightarrow E$ defined by

$$
\varphi\left(t_{1}, \ldots, t_{s} ; x\right)=\sum_{i=1}^{s} t_{i} e_{i}(x) .
$$

Hence for every $\mathbf{t}=\left(t_{1}, \ldots, t_{s}\right) \in R^{s}$ we have an embedding $\varphi_{\mathrm{t}}: X \rightarrow E$, given by fixing t. Let $X$ have a Riemann metric $g_{X}$. Then there exists a Riemann metric $g_{E}$ on $E$ such that

(i) for every $\mathbf{t} \in R^{s}, \varphi_{\mathbf{t}}: X \rightarrow E$ is an isometric embedding;

(ii) for every $\mathbf{t} \in R^{s}, \varphi_{\mathbf{t}}(X) \subseteq E$ is a totally geodesic submanifold. 
Proof. Endow $R^{s}$ with its usual Euclidean inner product. Then give $R^{s} \times X$ the product metric. Therefore $i_{\mathrm{t}}: X \rightarrow(\mathrm{t}, X)$ is an isometric embedding and $(\mathbf{t}, X)$ is a totally geodesic submanifold of $R^{s} \times X$. We know that $\varphi: R^{s} \times X \rightarrow E$ is an embedding, hence we can extend the metric on $\varphi\left(R^{s} \times X\right)$ to all of $E$, so that $\varphi$ is an isometric embedding with respect to this metric. Denote this Riemann metric by $\bar{g}_{E}$.

$\varphi: R^{s} \times X \rightarrow E$ is a vector bundle homomorphism over $X$. Let us look at the short exact sequence

$$
0 \longrightarrow R^{s} \times X \underset{\eta}{\stackrel{\varphi}{\rightleftarrows}} E \underset{\xi}{\stackrel{\lambda}{\rightleftarrows}} \operatorname{coker}(\varphi) \longrightarrow 0
$$

of vector bundles over $X$. Let $\eta: E \rightarrow R \times X$ be a splitting of this sequence, and let $\xi$ be the induced "splitting on the right." Hence

$$
i d_{E}=\varphi \eta+\xi \lambda
$$

Let $\bar{A}$ : coker $(\varphi) \rightarrow \operatorname{coker}(\varphi)$ be the antipodal map on the fibers of coker $(\varphi)$. Let us define the diffeomorphism $A: E \rightarrow E$ as follows: let $v \in E_{x}$, and define

$$
A v=\varphi \eta(v)-\xi \lambda(v)
$$

Hence $A^{2}=i d_{E}$ and this defines an action of $Z_{2}$ on $E$ which has $R^{s} \times X$ as its manifold of fixed points. We can now define a new metric $g_{E}$ on $E$ by the formula

$$
g_{E}(u, v)=\frac{\bar{g}_{E}(u, v)+\bar{g}_{E}(d A(u), d A(v))}{2}
$$

for any two vector fields $u$ and $v$ on $E$. Hence $A$ is an isometry with respect to the metric $g_{E}$. However $A$ left $X \times R^{s}$ pointwise fixed, therefore the new metric $g_{E}$ agrees with the old metric $\bar{g}_{E}$ on vectors tangent to $R^{s} \times X$. Hence $\varphi_{\mathrm{t}}: X \rightarrow E$ is an isometric embedding for each $\mathbf{t} \in R^{s}$.

It is a classical theorem in Riemannian geometry that the submanifold of fixed points of a group of isometries on a Riemannian manifold is a totally geodesic submanifold. Furthermore if $X$ is a submanifold of $Y$ and $Y$ is a submanifold of $Z$, and if $X$ is totally geodesic in $Y$ and $Y$ is totally geodesic in $Z$, then $X$ is totally geodesic in $Z$. Hence $\varphi_{\mathrm{t}}(X) \subseteq E$ is also a totally geodesic submanifold for all $\mathbf{t} \in R^{s}$.

Corollary 7.7. Let $i: X \rightarrow Y$ be an embedding of a manifold $X$ in a manifold $Y$. Let $N_{i, Y}(X)$ be the normal bundle of $i$, and let $e_{1}, \ldots, e_{s}$ be $s$ linearly independent cross-sections of $N_{i, Y}(X)$. Let $g_{X}$ be a given Riemann metric on $X$. Then there exists a neighborhood $U$ of $o \in R^{s}$, and an embedding $\varphi: U \times X \rightarrow Y$, and a Riemann metric 
$g_{Y}$ on $Y$ with the following properties. Let $\varphi_{\mathbf{t}}: X \rightarrow Y$ denote the embedding $\varphi_{\mathbf{t}}(x)=\varphi(\mathbf{t}, x)=\varphi\left(t_{1}, \ldots, t_{s} ; x\right)$ for a fixed $\mathbf{t} \in U$, and for every $x \in X$.

(i) $\varphi_{\mathrm{t}}$ is an isometric embedding for every $\mathbf{t} \in U$.

(ii) $\varphi_{\mathbf{t}}(X)$ is a totally geodesic submanifold for every $\mathbf{t} \in U$.

(iii) Let $\partial / \partial t_{1} \cdots \partial / \partial t_{s}$ be the usual basis for the vector fields on $R^{s}$ given by picking global coordinates $\left(t_{1}, \ldots, t_{s}\right)$. Let $e_{i}(x)=d \varphi_{(0 \times x)}\left(\partial / \partial t_{i}\right)$ for $i=1,2, \ldots, s$. Then the set of $s$ vector fields $e_{i}(x)$ on $i(X)$ are mutually perpendicular and normal to $i(X)$.

Proof. Let $i: X \rightarrow Y$ be the embedding. Let $T_{i, Y}(X)$ be the "open" tubular neighborhood of the embedding $i . T_{i, Y}(X) \subseteq Y$ and we know it is diffeomorphic to the normal bundle $N_{i, Y}{ }^{i}(X)$. Hence we can apply the second proposition to get the desired metric on $T_{i, Y}(X)$. The final result is obtained by applying the section extension theorem to any "closed tubular neighborhood" contained in $T_{i, Y}(X)$.

The above corollary with $s$ set equal to zero gives us the following result.

Corollary 7.8. Let $i: X \rightarrow Y$ be an embedding. Let $g_{X}$ be a Riemann metric on $X$. Then there exists a Riemann metric $g_{Y}$ on $Y$ such that:

(i) $i$ is an isometric embedding,

(ii) $i(X)$ is a totally geodesic submanifold.

\section{BIBLIOGRAPHY}

1. A. Borel and J. C. Moore, Homology theory for locally compact spaces, Michigan Math. J. 7 (1960), 137-159.

2. A. Borel and A. Haefliger, La classe d'homologie fundamentale d'un espace analytique, Bull. Soc. Math. France 89 (1961), 461-513.

3. H. Whitney, Elementary structure of real algebraic varieties, Ann. of Math. (2) 66 (1957), 545-556.

4. E. A. Feldman, Geometry of immersions. I, Trans. Amer. Math. Soc. 120 (1965), 185-224.

5. W. F. Pohl, Differential geometry of higher order, Topology 1 (1962), 169-211.

6. R. Thom (notes by H. Levine), Singularities of differentiable mappings, Mimeographed notes, Bonn Univ., Bonn, 1959.

7. H. Whitney, Local properties of analytic varieties, Differential and Combinatorial Topology, Princeton Univ. Press, Princeton, N. J., 1964.

8. I. R. Porteous, Simple singularities of maps, Mimeographed notes, Columbia Univ., New York, 1962.

9. J. Milnor, Differential topology, Mimeographed notes, Princeton Univ., Princeton, N. J., 1959.

10. D. J. Struik, Differential geometry, Addison-Wesley, Reading, Mass., 1950.

11. $\mathrm{H}$. Hopf, Lectures on differential geometry in the large, Mimeographed notes, Stanford Univ., Stanford, Calif., 1956.

12. A. Haefliger and A. Kosinski, Un théorème de Thom sur les singularités des applications différentiables, Séminaire Henri Cartan (1956/1957), Exposé 8, Secrétariat mathématique, Paris, 1958.

13. R. Abraham, Lectures of Smale on differential topology, Mimeographed notes, Columbia Univ., New York, 1963. 
14. N. E. Steenrod, Topology of fiber bundles, Princeton Univ. Press, Princeton, N. J., 1951.

15. J. L. Koszul, Lectures on fiber bundles and differential geometry, Tata Institute of Fundamental Research, Bombay, 1960.

16. H. W. Whitney, Differentiable manifolds, Ann. of Math. (2) 37 (1936), 645-680.

17. E. A. Feldman, Geometry of immersions. II, Bull. Amer. Math. Soc. 70 (1964), 600-607.

18. F. Hirzebruch, Neue topologische methoden in der algebraischen geometrie, SpringerVerlag, Berlin, 1956.

\section{COLUMBia UNIVERSITY,}

New YoRK, New YoRK

PrinCETON UNIVERSITY,

Princeton, New JeRsey 